This is a self-archived version of an original article. This version may differ from the original in pagination and typographic details.

Author(s): Ardentov, Andrei A.; Le Donne, Enrico; Sachkov, Yuri L.

Title: Sub-Finsler Geodesics on the Cartan Group

Year: 2019

Version: Accepted version (Final draft)

Copyright: (c) 2019, Pleiades Publishing, Ltd.

Rights: In Copyright

Rights url: http://rightsstatements.org/page/InC/1.0/?language=en

Please cite the original version:

Ardentov, A. A., Le Donne, E., \& Sachkov, Y. L. (2019). Sub-Finsler Geodesics on the Cartan Group. Regular and Chaotic Dynamics, 24(1), 36-60.

https://doi.org/10.1134/S1560354719010027 


\title{
Sub-Finsler geodesics on the Cartan group*
}

\author{
A.Ardentov ${ }^{\dagger}$ E. Le Donne ${ }^{\ddagger}$ Yu. Sachkov ${ }^{\S}$
}

October 15, 2018

\begin{abstract}
This paper is a continuation of the work by the same authors on the Cartan group equipped with the subFinsler $\ell_{\infty}$ norm. We start by giving a detailed presentation of the structure of bang-bang extremal trajectories. Then we prove upper bounds on the number of switchings on bang-bang minimizers. We prove that any normal extremal is either bang-bang, or singular, or mixed. Consequently, we study mixed extremals. In particular, we prove that every two points can be connected by a piecewise smooth minimizer, and we give a uniform bound on the number of such pieces.
\end{abstract}

\section{Introduction}

There are several motivations for studying sub-Finsler geometry on Lie groups, especially in geometric group theory and in harmonic analysis. We only mention the prominent articles [10, 6, 4] and then we refer to the introductions of [15, 17] for a broad explanation of the reasons and for several references of the state-of-the-art.

On the one hand, as in sub-Riemannian geometry, distributions of step 2 are easier to study and there is already some good understanding of the lower dimensional cases, see [15]. On the other hand, sub-Finsler structures defined by smooth norms have a similar theory that in the sub-Riemannian case. For these reasons the challenge is to study step-3 sub-Finsler groups with a non-strictly convex norm. The lower dimensional examples are the Engel group and the Cartan group, which both have step 3 and rank 2.

In this paper we study the Cartan group, since it is the free-nilpotent group of rank 2 and step 3 (so the Engel group is a quotient of this group), equipped with the $\ell_{\infty}$ sub-Finsler structure. In our previous paper [17, adopting the point of view of time-optimal control theory, we characterized extremal curves via Pontryagin maximum principle, we described abnormal and singular arcs, and we constructed the bang-bang flow.

The Cartan distribution can be expressed by the span of two vector fields $X_{1}, X_{2}$. We consider the $\ell_{\infty}$ norm with respect to $X_{1}, X_{2}$. Hence, every admissible trajectory is characterized by two controls. A summary of the results of this paper is given by the following statements.

Theorem 1. In the $\ell_{\infty}$ sub-Finsler structure on the Cartan group the length-minimizing trajectories are of three not-mutually-exclusive types:

(i) one component of the control is constantly equal to 1 or -1 ,

(ii) bang-bang trajectory,

(iii) piecewise smooth concatenation of trajectories of types (i) and (ii).

The length-minimizers that are of type (ii) but not of type (i) have at most 12 arcs. The length-minimizers of type (iii) have at most 14 arcs. All curves of type (i) are length-minimizers. Moreover, for every trajectory of type (i) there exists a piecewise-smooth length-minimizing trajectory connecting the same two points and having at most 5 smooth pieces.

${ }^{*}$ Sections 1, 2 and 6 of the paper are written by E. Le Donne, and Sections 3-5 and 7, 8 are written by A. Ardentov and Yu. Sachkov. The work of A. Ardentov and Yu. Sachkov is supported by the Russian Science Foundation under grant 17-11-01387 and performed in Ailamazyan Program Systems Institute of Russian Academy of Sciences. E. Le Donne was partially supported by the Academy of Finland (grant 288501 'Geometry of subRiemannian groups') and by the European Research Council (ERC Starting Grant 713998 GeoMeG 'Geometry of Metric Groups').

${ }^{\dagger}$ Program Systems Institute, Pereslavl-Zalessky, Russia, aaa@pereslavl.ru

$\ddagger$ Department of Mathematics and Statistics, P.O. Box 35, FI-40014, University of Jyväskylä, Finland, ledonne@msri.org

$\S$ Program Systems Institute, Pereslavl-Zalessky, Russia, yusachkov@gmail.com 
As a corollary, we deduce that any pair of points can be connected by an optimal piecewise-smooth trajectory with at most 14 arcs.

The paper has the following structure. In Sec. 2 we recall the problem statement and the main results on it obtained in previous paper [17. Section 3 is devoted to detailed study of structure of bang-bang extremal trajectories implied by Pontryagin Maximum Principle. In Sec. 4 we prove upper bounds on the number of switchings on bangbang minimizers. In Sec. 5 we prove that any normal extremal is either bang-bang, or singular, or mixed. Further, Sec. 6 is devoted to the study of mixed extremals, including upper bound on the number of switchings. Finally, in Sec. 7 we obtain a uniform bound on the number of smooth pieces on minimizers connecting arbitrary points in the Cartan group.

\section{Problem statement and previous results}

Consider the 5-dimensional free nilpotent Lie algebra with 2 generators, of step 3 . There exists a basis $L=$ $\operatorname{span}\left(X_{1}, \ldots, X_{5}\right)$ in which the product rule in $L$ takes the form

$$
\left[X_{1}, X_{2}\right]=X_{3}, \quad\left[X_{1}, X_{3}\right]=X_{4}, \quad\left[X_{2}, X_{3}\right]=X_{5}, \quad \text { ad } X_{4}=\operatorname{ad} X_{5}=0 .
$$

The Lie algebra $L$ is called the Cartan algebra, and the corresponding connected simply connected Lie group $M$ is called the Cartan group. We will use the following model:

$$
M=\mathbb{R}_{x, y, z, v, w}^{5}
$$

with the Lie algebra $L$ modeled by left-invariant vector fields on $\mathbb{R}^{5}$

$$
\begin{aligned}
& X_{1}=\frac{\partial}{\partial x}-\frac{y}{2} \frac{\partial}{\partial z}-\frac{x^{2}+y^{2}}{2} \frac{\partial}{\partial w}, \\
& X_{2}=\frac{\partial}{\partial y}+\frac{x}{2} \frac{\partial}{\partial z}+\frac{x^{2}+y^{2}}{2} \frac{\partial}{\partial v}, \\
& X_{3}=\frac{\partial}{\partial z}+x \frac{\partial}{\partial v}+y \frac{\partial}{\partial w}, \\
& X_{4}=\frac{\partial}{\partial v} \\
& X_{5}=\frac{\partial}{\partial w} .
\end{aligned}
$$

The product rule in the Cartan group $M$ in this model is given in [11.

Left-invariant $\ell_{\infty}$ sub-Finsler problem on the Cartan group is stated as the following time-optimal problem:

$$
\begin{aligned}
& \dot{q}=u_{1} X_{1}+u_{2} X_{2}, \quad q \in M, \quad u \in U=\left\{u \in \mathbb{R}^{2} \mid\|u\|_{\infty} \leq 1\right\} \\
& \|u\|_{\infty}=\max \left(\left|u_{1}\right|,\left|u_{2}\right|\right), \\
& q(0)=q_{0}=\operatorname{Id}=(0, \ldots, 0), \quad q(T)=q_{1}, \\
& T \rightarrow \min .
\end{aligned}
$$

Problem 2.1 - 2.3) was considered first in paper [17. We recall the main results of that paper.

Existence of optimal controls follows from Rashevsky-Chow and Filippov theorem [12].

Pontryagin Maximum Principle implies that optimal abnormal controls are constant.

Introduce linear-on-fibers Hamiltonians $h_{i}(\lambda)=\left\langle\lambda, X_{i}\right\rangle, \lambda \in T^{*} M, i=1, \ldots, 5$. A normal extremal arc $\lambda_{t}, t \in I=(\alpha, \beta) \subset[0, T]$ is called:

- a bang-bang arc if

$$
\operatorname{card}\left\{t \in I \mid h_{1} h_{2}\left(\lambda_{t}\right)=0\right\}<\infty,
$$

- a singular arc if one of the condition holds:

$$
\begin{array}{ll}
h_{1}\left(\lambda_{t}\right) \equiv 0, \quad t \in I \quad\left(h_{1} \text {-singular arc }\right), \text { or } \\
h_{2}\left(\lambda_{t}\right) \equiv 0, \quad t \in I \quad\left(h_{2} \text {-singular arc }\right),
\end{array}
$$

- a mixed arc if it consists of a finite number of bang-bang and singular arcs. 
Singular controls have one of components constantly equal to 1 or -1 , thus they are optimal. The fix-time attainable set along singular trajectories was explicitly described and was shown to be semi-algebraic.

Bang-bang extremal trajectories satisfy the Hamiltonian system with the Hamiltonian function $H=\left|h_{1}\right|+\left|h_{2}\right|$ :

$$
\left\{\begin{array}{l}
\dot{h}_{1}=-s_{2} h_{3}, \\
\dot{h}_{2}=s_{1} h_{3}, \\
\dot{h}_{3}=s_{1} h_{4}+s_{2} h_{5}, \\
\dot{h}_{4}=\dot{h}_{5}=0, \\
\dot{q}=s_{1} X_{1}+s_{2} X_{2} .
\end{array}\right.
$$

The dual of the Lie algebra $L^{*}=T_{\mathrm{Id}}^{*} M$ has Casimir functions $h_{4}, h_{5}, E=\frac{h_{3}^{2}}{2}+h_{1} h_{5}-h_{2} h_{4}$, thus Hamiltonian system 2.4 has integrals $h_{4}, h_{5}, E$, and $H$.

The mapping $(\lambda, q) \mapsto(k \lambda, q), k>0$, preserves extremal trajectories, thus we can consider only the reduced case

$$
H(\lambda) \equiv 1
$$

With the use of the coordinate $\theta \in S^{1}=\mathbb{R} / 2 \pi \mathbb{Z}$ :

$$
h_{1}=\operatorname{sgn}(\cos \theta) \cos ^{2} \theta, \quad h_{2}=\operatorname{sgn}(\sin \theta) \sin ^{2} \theta,
$$

the vertical part of Hamiltonian system (2.4) reduces to the following system:

$$
\left\{\begin{array}{l}
\dot{\theta}=\frac{h_{3}}{|\sin 2 \theta|}, \quad \theta \neq \frac{\pi n}{2}, \\
\dot{h}_{3}=s_{1} h_{4}+s_{2} h_{5}, \quad s_{1}=\operatorname{sgn} \cos \theta, \quad s_{2}=\operatorname{sgn} \sin \theta
\end{array}\right.
$$

System 2.5 is preserved by the group of symmetries of the square $\left\{\left(h_{1}, h_{2}\right) \in \mathbb{R}^{2}|| h_{1}|+| h_{2} \mid=1\right\}$. Thus in the study of system (2.5) we can restrict ourselves by the case $h_{4} \geq h_{5} \geq 0$. This case obviously decomposes into the following sub-cases:

1) $h_{4}>h_{5}>0$

2) $h_{4}>h_{5}=0$,

3) $h_{4}=h_{5}>0$,

4) $h_{4}=h_{5}=0$.

\section{$3 \quad$ Structure of bang-bang trajectories}

In this section we consider, case by case, the structure of bang-bang trajectories implied by Pontryagin Maximum Principle.

\subsection{Case 1)}

Let $h_{4}>h_{5}>0$. Then system (2.5) has the phase portrait given in Fig. 1, see Subsubsec. 7.2.1 [17].

The domain $\left\{\lambda \in C \mid h_{4}>h_{5}>0\right\}$ of the cylinder $C=L^{*} \cap\{H=1\}$ admits a decomposition defined by the energy integral $E$ :

$$
\begin{aligned}
& \left\{\lambda \in C \mid h_{4}>h_{5}>0\right\}=\cup_{i=1}^{8} C_{i}, \\
& C_{1}=E^{-1}\left(-h_{4}\right), \quad C_{2}=E^{-1}\left(-h_{4},-h_{5}\right), \quad C_{3}=E^{-1}\left(-h_{5}\right), \quad C_{4}=E^{-1}\left(-h_{5}, h_{5}\right), \\
& C_{5}=E^{-1}\left(h_{5}\right), \quad C_{6}=E^{-1}\left(h_{5}, h_{4}\right), \quad C_{7}=E^{-1}\left(h_{4}\right), \quad C_{8}=E^{-1}\left(h_{4},+\infty\right) .
\end{aligned}
$$

\subsubsection{Case 1), level set $C_{1}$}

Let $h_{4}>h_{5}>0, E=-h_{4}$. Then $\theta \equiv \frac{\pi}{2}, h_{3} \equiv 0$. Thus $h_{1}\left(\lambda_{t}\right) \equiv 0, h_{2}\left(\lambda_{t}\right) \equiv 1$, i.e., the extremal $\lambda_{t}$ is $h_{1}$-singular and not bang-bang. 


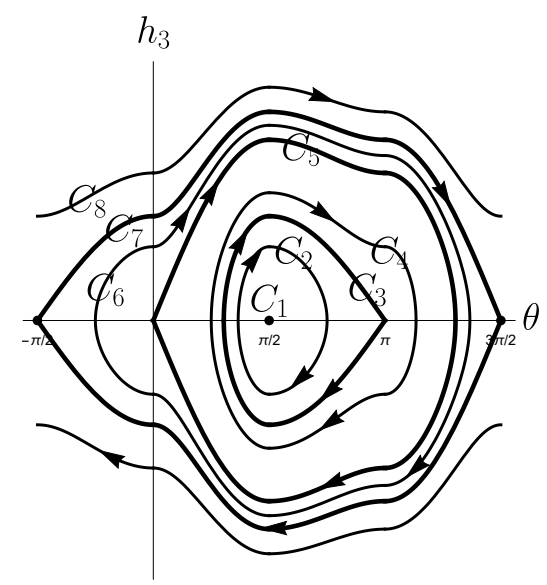

Figure 1: Phase portrait of system 2.5 in case 1 )

\subsubsection{Case 1), domain $C_{2}$}

Let $h_{4}>h_{5}>0, E \in\left(-h_{4},-h_{5}\right)$. The corresponding bang-bang extremal is shown in Figs. 2, 3 .

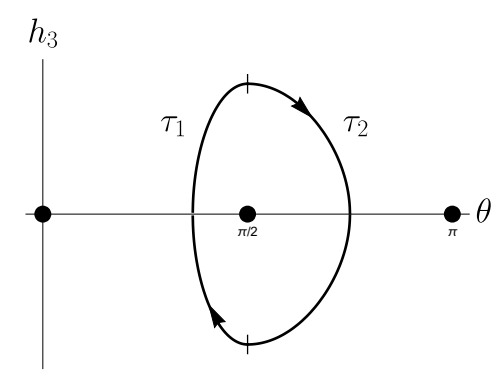

Figure 2: $\left(\theta(t), h_{3}(t)\right)$ : Case 1), domain $C_{2}$

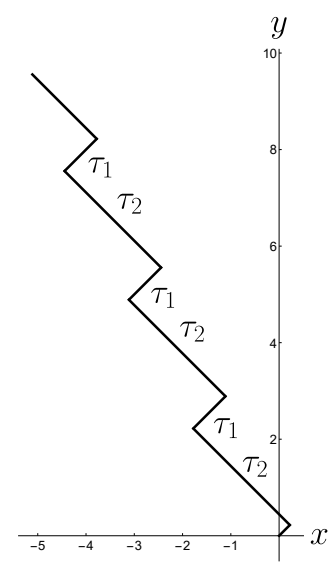

Figure 3: $(x(t), y(t))$ : Case 1), domain $C_{2}$

Denote by $\tau_{1}, \tau_{2}$, the time intervals between successive switchings of control:

$$
\begin{array}{lll}
\theta(0)=\frac{\pi}{2}, & \theta(t) \in\left(0, \frac{\pi}{2}\right) \quad \text { for } \quad t \in\left(0, \tau_{1}\right), & \theta\left(\tau_{1}\right)=\frac{\pi}{2}, \\
\theta(0)=\frac{\pi}{2}, & \theta(t) \in\left(\frac{\pi}{2}, \pi\right) \quad \text { for } \quad t \in\left(0, \tau_{2}\right), & \theta\left(\tau_{2}\right)=\frac{\pi}{2} .
\end{array}
$$

Compute the interval $\tau_{1}$ via the Casimirs $E, h_{4}, h_{5}$ :

$$
\begin{aligned}
& \theta \in\left[0, \frac{\pi}{2}\right] \Rightarrow E=\frac{h_{3}^{2}}{2}+h_{5} \cos ^{2} \theta-h_{4} \sin ^{2} \theta \\
& \theta=\frac{\pi}{2} \Rightarrow E=\frac{h_{3}^{2}}{2}-h_{4}, \quad h_{3}= \pm \sqrt{2\left(E+h_{4}\right)} \\
& h_{3}(0)=-\sqrt{2\left(E+h_{4}\right)}, \quad h_{3}\left(\tau_{1}\right)=\sqrt{2\left(E+h_{4}\right)} \\
& h_{3}(t)=h_{3}(0)+\left(h_{4}+h_{5}\right) t \\
& \tau_{1}=\frac{h_{3}\left(\tau_{1}\right)-h_{3}(0)}{h_{4}+h_{5}}=\frac{2 \sqrt{2\left(E+h_{4}\right)}}{h_{4}+h_{5}}
\end{aligned}
$$


We compute similarly $\tau_{2}=\frac{2 \sqrt{2\left(E+h_{4}\right)}}{h_{4}-h_{5}}>\tau_{1}$. The rule of switchings of controls $u_{i}(t)=s_{i}=\operatorname{sgn} h_{i}(t), i=1,2$, follows from the phase portrait in Fig. 2. Thus we obtain a general form of a bang-bang trajectory in the case 1), $C_{2}$.

Proposition 1. In case 1$)$, domain $C_{2}: h_{4}>h_{5}>0, E \in\left(-h_{4},-h_{5}\right)$, a bang-bang control is obtained by choosing a finite segment of the following two-side infinite periodic sequence:

$$
\begin{array}{ccccccc}
\left(u_{1}, u_{2}\right): & \ldots & (+,+) & (-,+) & (+,+) & (-,+) & \ldots \\
& \tau_{1} & \tau_{2} & \tau_{1} & \tau_{2} &
\end{array}
$$

Duration of all segments of constancy of controls (except the first and the last ones) is equal to

$$
\tau_{1}=\frac{2 \sqrt{2\left(E+h_{4}\right)}}{h_{4}+h_{5}}, \quad \tau_{2}=\frac{2 \sqrt{2\left(E+h_{4}\right)}}{h_{4}-h_{5}}>\tau_{1} .
$$

The first and the last segments may take arbitrary values in the corresponding intervals $\left(0, \tau_{i}\right]$.

The subsequent analysis of the structure of bang-bang trajectories is completely analogous to the preceding one, thus we omit analogous computations and arguments in the following subsubsections.

\subsubsection{Case 1), level line $C_{3}$}

Let $h_{4}>h_{5}>0, E=-h_{5}$, the corresponding extremal is shown in Figs. 4 , 5 .
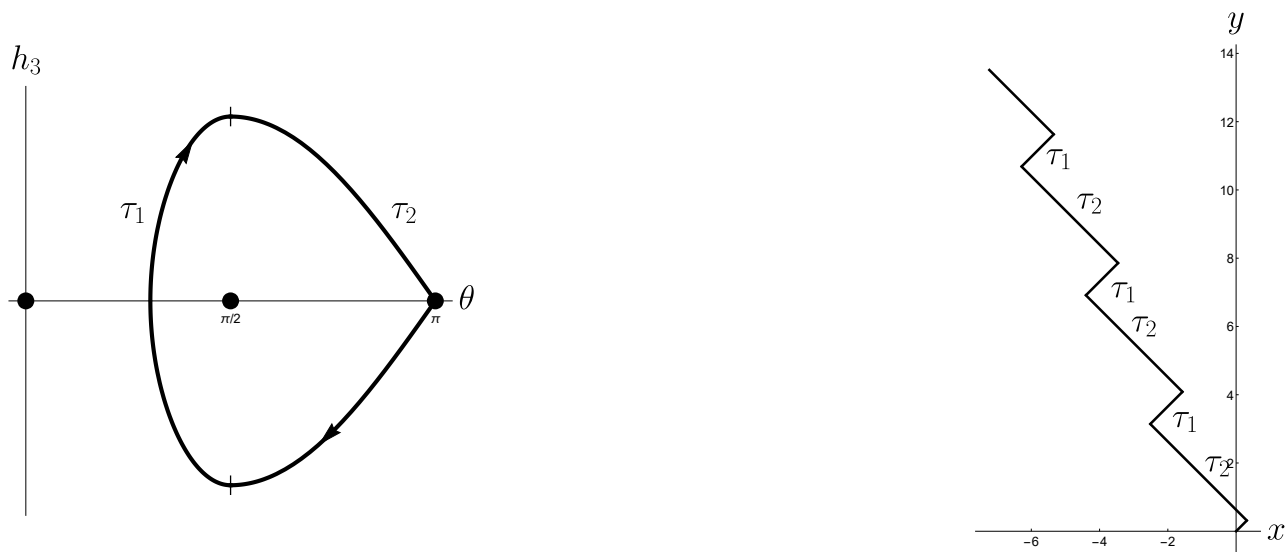

Figure 4: $\left(\theta(t), h_{3}(t)\right)$ : Case 1), level line $C_{3}$

Figure 5: $(x(t), y(t))$ : Case 1), level line $C_{3}$

Then the control has the form

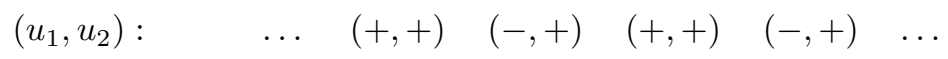

$$
\begin{array}{llll}
\tau_{1} & \tau_{2} & \tau_{1} & \tau_{2}
\end{array}
$$

with

$$
\tau_{1}=\frac{2 \sqrt{2\left(h_{4}-h_{5}\right)}}{h_{4}+h_{5}}, \quad \tau_{2}=\frac{2 \sqrt{2\left(h_{4}-h_{5}\right)}}{h_{4}-h_{5}}=2 \sqrt{\frac{2}{h_{4}-h_{5}}}>\tau_{1} .
$$

Notice that despite the fact that $h_{2}\left(\lambda_{t}\right)$ vanishes when $\theta=\pi, h_{3}=0$, the control $u_{2}(t)$ does not switch at such points since $h_{2}\left(\lambda_{t}\right)$ preserves sign near these points.

\subsubsection{Case 1), domain $C_{4}$}

Let $h_{4}>h_{5}>0, E \in\left(-h_{5}, h_{5}\right)$, the corresponding trajectory is shown in Figs. 6. 7 


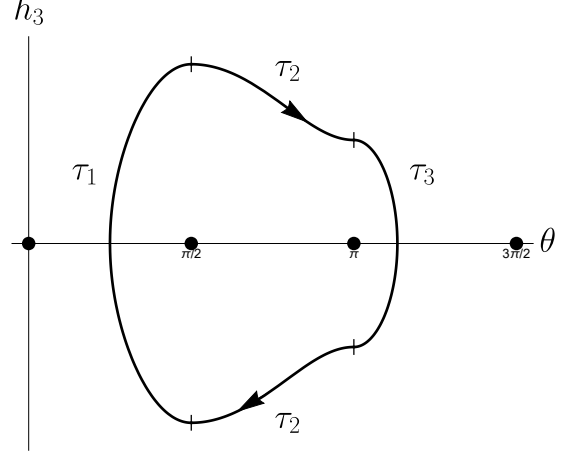

Figure 6: $\left(\theta(t), h_{3}(t)\right)$ : Case 1), domain $C_{4}$

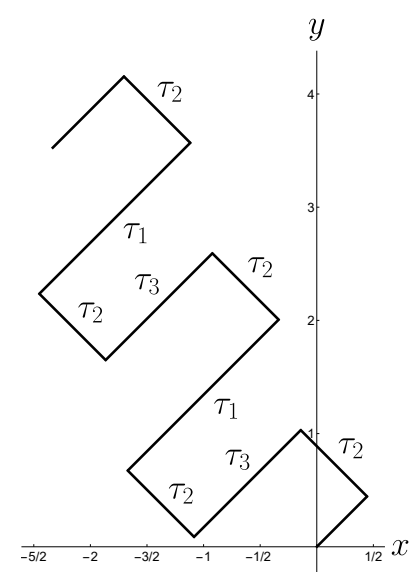

Figure 7: $(x(t), y(t))$ : Case 1), domain $C_{4}$

Then the control has the form:

$$
\begin{array}{cccccccc}
\left(u_{1}, u_{2}\right): & \cdots & (+,+) & (-,+) & (-,-) & (-,+) & (+,+) & \ldots \\
& & \tau_{1} & \tau_{2} & \tau_{3} & \tau_{2} & \tau_{1} &
\end{array}
$$

with

$$
\begin{gathered}
\tau_{1}=\frac{2 \sqrt{2\left(E+h_{4}\right)}}{h_{4}+h_{5}}, \quad \tau_{2}=\frac{\sqrt{2\left(E+h_{4}\right)}-\sqrt{2\left(E+h_{5}\right)}}{h_{4}-h_{5}}=\frac{2}{\sqrt{2\left(E+h_{4}\right)}+\sqrt{2\left(E+h_{5}\right)}}, \\
\tau_{3}=\frac{2 \sqrt{2\left(E+h_{5}\right)}}{h_{4}+h_{5}}<\tau_{1} .
\end{gathered}
$$

\subsubsection{Case 1), level line $C_{5}$}

Let $h_{4}>h_{5}>0, E=h_{5}$, the corresponding trajectory is shown in Figs. 8, 9 .

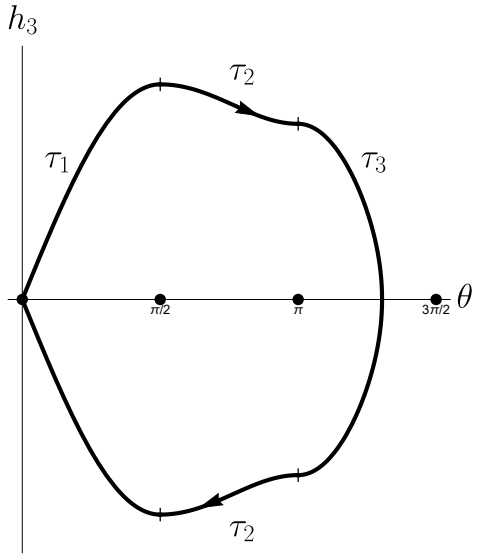

Figure 8: $\left(\theta_{t}, h_{3}(t)\right)$ : Case 1), level line $C_{5}$

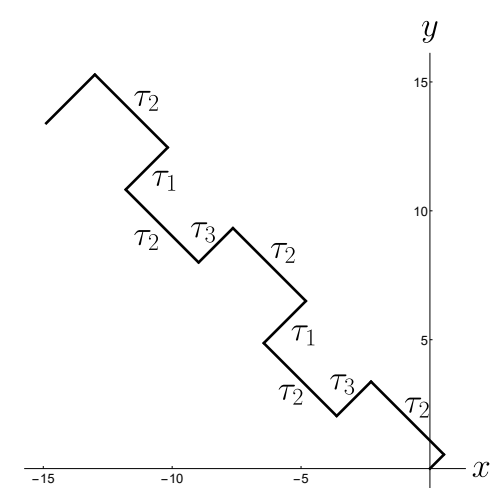

Figure 9: $(x(t), y(t)):$ Case 1), level line $C_{5}$

Then the control has the form:

$$
\begin{array}{cccccccc}
\left(u_{1}, u_{2}\right): & \cdots & (+,+) & (-,+) & (-,-) & (-,+) & (+,+) & \ldots \\
& \tau_{1} & \tau_{2} & \tau_{3} & \tau_{2} & \tau_{1} &
\end{array}
$$


with

$$
\tau_{1}=2 \sqrt{\frac{2}{h_{4}+h_{5}}}, \quad \tau_{2}=\frac{\sqrt{2\left(h_{4}+h_{5}\right)}-2 \sqrt{h_{5}}}{h_{4}-h_{5}}, \quad \tau_{3}=\frac{4 \sqrt{h_{5}}}{h_{4}+h_{5}} .
$$

The control $u_{1}(t)$ does not switch when $\theta=0, h_{3}=0$.

\subsubsection{Case 1), domain $C_{6}$}

Let $h_{4}>h_{5}>0, E \in\left(h_{5}, h_{4}\right)$, the corresponding trajectory is shown in Figs. 10,11

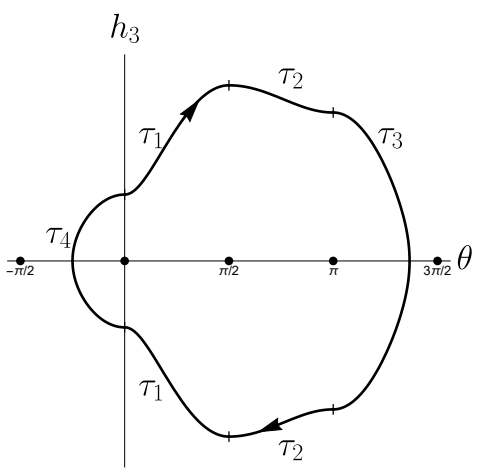

Figure 10: $\left(\theta(t), h_{3}(t)\right)$ : Case 1), domain $C_{6}$

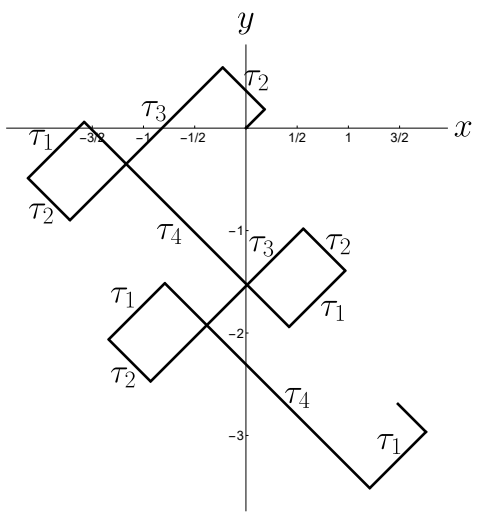

Figure 11: $(x(t), y(t))$ : Case 1), domain $C_{6}$

Then the control has the form:

$$
\begin{array}{cccccccccc}
\left(u_{1}, u_{2}\right): & \cdots & (+,+) & (-,+) & (-,-) & (-,+) & (+,+) & (+,-) & (+,+) & \ldots \\
& & \tau_{1} & \tau_{2} & \tau_{3} & \tau_{2} & \tau_{1} & \tau_{4} & \tau_{1} &
\end{array}
$$

with

$$
\begin{gathered}
\tau_{1}=\frac{\sqrt{2\left(E+h_{4}\right)}-\sqrt{2\left(E-h_{5}\right)}}{h_{4}+h_{5}}=\frac{2}{\sqrt{2\left(E+h_{4}\right)}+\sqrt{2\left(E-h_{5}\right)}} \\
\tau_{2}=\frac{\sqrt{2\left(E+h_{4}\right)}-\sqrt{2\left(E+h_{5}\right)}}{h_{4}-h_{5}}=\frac{2}{\sqrt{2\left(E+h_{4}\right)}+\sqrt{2\left(E+h_{5}\right)}} \\
\tau_{3}=\frac{2 \sqrt{2\left(E+h_{5}\right)}}{h_{4}+h_{5}}, \quad \tau_{4}=\frac{2 \sqrt{2\left(E-h_{5}\right)}}{h_{4}-h_{5}}
\end{gathered}
$$

\subsubsection{Case 1), level line $C_{7}$}

Let $h_{4}>h_{5}>0, E=h_{4}$, the corresponding phase portrait is shown in Fig. 12 . 


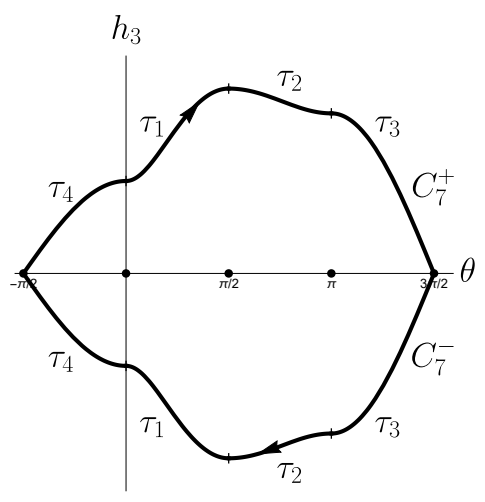

Figure 12: $\left(\theta(t), h_{3}(t)\right)$ : Case 1), level line $C_{7}$

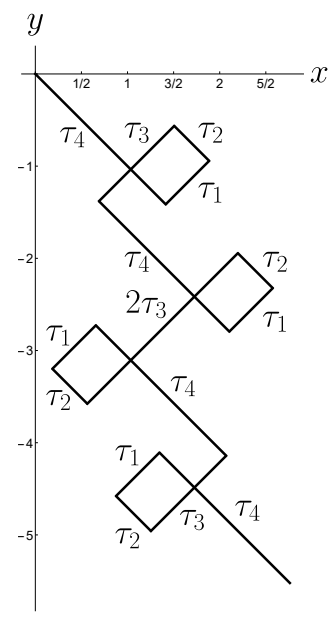

Figure 13: $(x(t), y(t))$ : Case 1), level line $C_{7}$

The level line $C_{7}$ is defined in the domains $\left\{\theta \in\left(-\frac{\pi}{2}, 0\right)\right\}$ and $\left\{\theta \in\left(\pi, \frac{3 \pi}{2}\right)\right\}$ by the equations

$$
h_{3}= \pm \sqrt{2\left(h_{4}-h_{5}\right)} \cos \theta \text { and } h_{3}= \pm \sqrt{2\left(h_{4}+h_{5}\right)} \cos 2 \theta .
$$

Thus the level line $C_{7}$ is homeomorphic to figure 8 , with self-intersection at the point $\left(\theta, h_{3}\right)=\left(\frac{3 \pi}{2}, 0\right)$. The intersections $C_{7}^{+}=C_{7} \cap\left\{h_{3} \geq 0\right\}, C_{7}^{-}=C_{7} \cap\left\{h_{3} \leq 0\right\}$ are continuous curves homeomorphic to $S^{1}$, with the only singularity - the corner point $\left(\theta, h_{3}\right)=\left(\frac{3 \pi}{2}, 0\right)$.

Each bang-bang control is obtained by choosing a finite segment from the following infinite periodic graph:

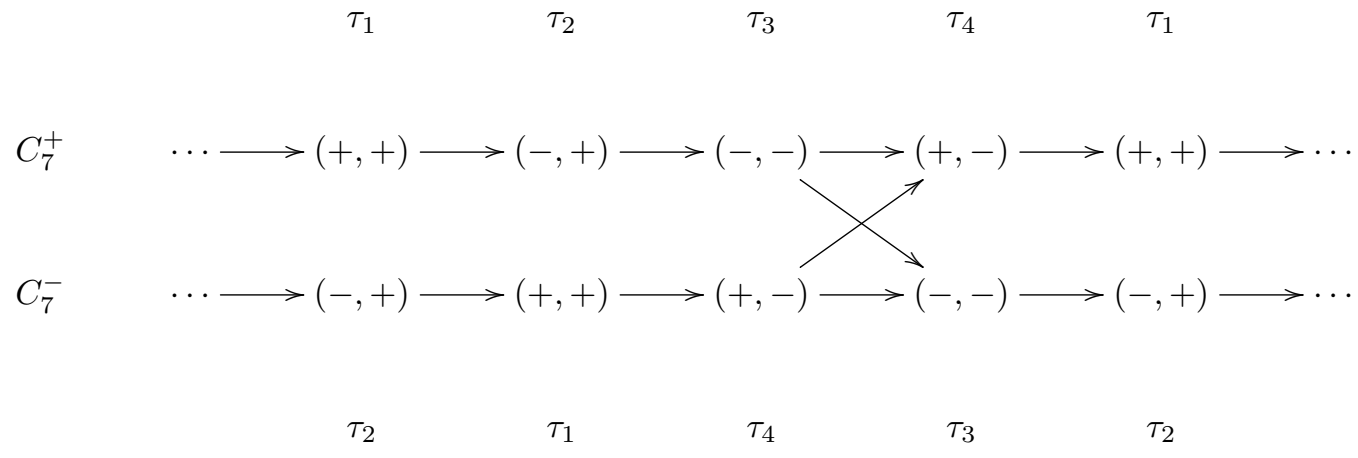

We have

$$
\begin{aligned}
\tau_{1}=\frac{2 \sqrt{h_{4}}-\sqrt{2\left(h_{4}-h_{5}\right)}}{h_{4}+h_{5}}, & \tau_{2}=\frac{2 \sqrt{h_{4}}-\sqrt{2\left(h_{4}+h_{5}\right)}}{h_{4}-h_{5}}, \\
\tau_{3}=\sqrt{\frac{2}{h_{4}+h_{5}}}, & \tau_{4}=\sqrt{\frac{2}{h_{4}-h_{5}}} .
\end{aligned}
$$

The curves $(x(t), y(t))$ corresponding to the curves $C_{7}^{+}$and $C_{7}^{-}$are shown in Figs. 14 and 15 respectively. An example of curve $(x(t), y(t))$ corresponding to two curves $C_{7}^{+}$and two curves $C_{7}^{-}$is given in Fig. 13 . 


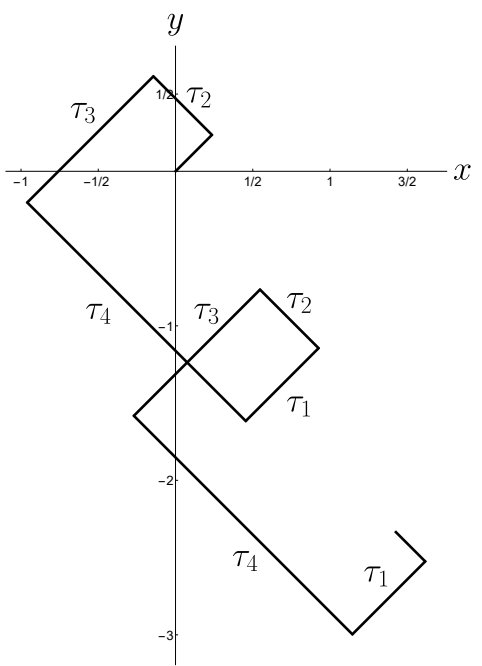

Figure 14: $(x(t), y(t))$ : Case 1), curve $C_{7}^{+}$

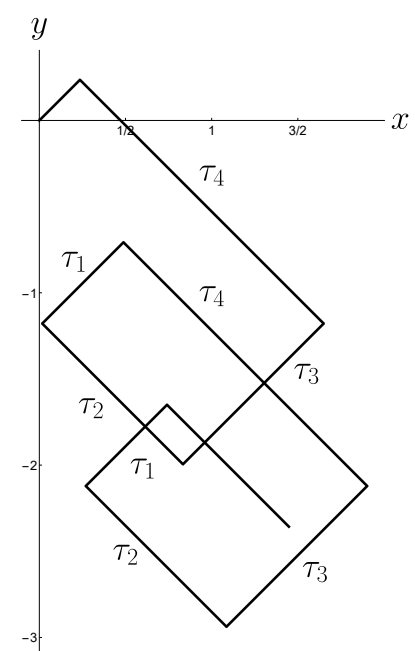

Figure 15: $(x(t), y(t)):$ Case 1), curve $C_{7}^{-}$

\subsubsection{Case 1), domain $C_{8}$}

Let $h_{4}>h_{5}>0, E>h_{4}$, the corresponding trajectory is shown in Figs. 16, 17 .

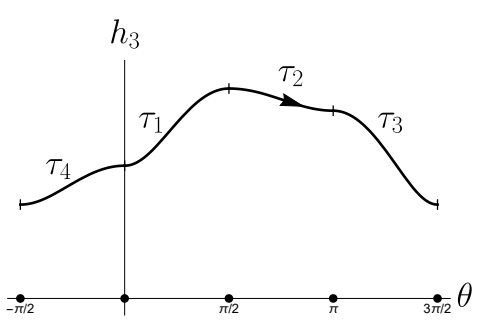

Figure 16: $\left(\theta(t), h_{3}(t)\right)$ : Case 1), domain $C_{8}$

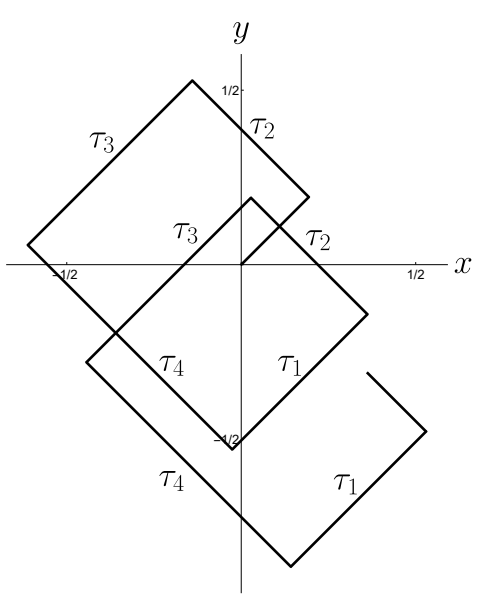

Figure 17: $(x(t), y(t))$ : Case 1), domain $C_{8}$

In the case $h_{3}>0$ the bang-bang control has the form

$$
\begin{array}{cccccccc}
\left(u_{1}, u_{2}\right): & \cdots & (+,+) & (-,+) & (-,-) & (+,-) & (+,+) & \ldots
\end{array}
$$

$\begin{array}{lllll}\tau_{1} & \tau_{2} & \tau_{3} & \tau_{4} & \tau_{1}\end{array}$ 
In the case $h_{3}<0$ the order of switchings is opposite. We have

$$
\begin{aligned}
& \tau_{1}=\frac{\sqrt{2\left(E+h_{4}\right)}-\sqrt{2\left(E-h_{5}\right)}}{h_{4}+h_{5}}=\frac{2}{\sqrt{2\left(E+h_{4}\right)}+\sqrt{2\left(E-h_{5}\right)}}, \\
& \tau_{2}=\frac{\sqrt{2\left(E+h_{4}\right)}-\sqrt{2\left(E+h_{5}\right)}}{h_{4}-h_{5}}=\frac{2}{\sqrt{2\left(E+h_{4}\right)}+\sqrt{2\left(E+h_{5}\right)}} \\
& \tau_{3}=\frac{\sqrt{2\left(E+h_{5}\right)}-\sqrt{2\left(E-h_{4}\right)}}{h_{4}+h_{5}}=\frac{2}{\sqrt{2\left(E+h_{5}\right)}+\sqrt{2\left(E-h_{4}\right)}}, \\
& \tau_{4}=\frac{\sqrt{2\left(E-h_{5}\right)}-\sqrt{2\left(E-h_{4}\right)}}{h_{4}-h_{5}}=\frac{2}{\sqrt{2\left(E-h_{5}\right)}+\sqrt{2\left(E-h_{4}\right)}} .
\end{aligned}
$$

\subsection{Case 2)}

Let $h_{4}>h_{5}=0$. Then system 2.5 has the phase portrait given in Fig. 18, see Subsubsec. 7.2.2 [17.

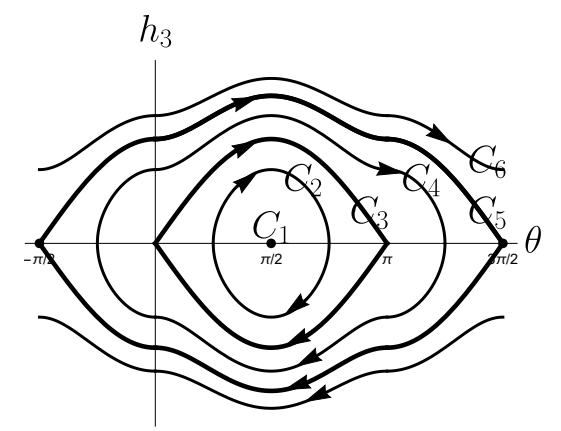

Figure 18: Phase portrait of system 2.5$)$ in case 2$)$

The domain $\left\{\lambda \in C \mid h_{4}>h_{5}=0\right\}$ of the cylinder $C=L^{*} \cap\{H=1\}$ admits a decomposition defined by the energy integral $E$ :

$$
\begin{aligned}
& \left\{\lambda \in C \mid h_{4}>h_{5}=0\right\}=\cup_{i=1}^{6} C_{i}, \\
& C_{1}=E^{-1}\left(-h_{4}\right), \quad C_{2}=E^{-1}\left(-h_{4}, 0\right), \quad C_{3}=E^{-1}(0), \\
& C_{4}=E^{-1}\left(0, h_{4}\right), \quad C_{5}=E^{-1}\left(h_{4}\right), \quad C_{6}=E^{-1}\left(h_{4},+\infty\right) .
\end{aligned}
$$

\subsubsection{Case 2), level set $C_{1}$}

Let $h_{4}>h_{5}=0, E=-h_{4}$. Then $\theta \equiv \frac{\pi}{2}, h_{3} \equiv 0$, and the corresponding trajectory is $h_{1}$-singular.

\subsubsection{Case 2), domain $C_{2}$}

We have $h_{4}>h_{5}=0, E \in\left(-h_{4}, 0\right)$, the corresponding trajectory is shown in Figs. 19,20 , 


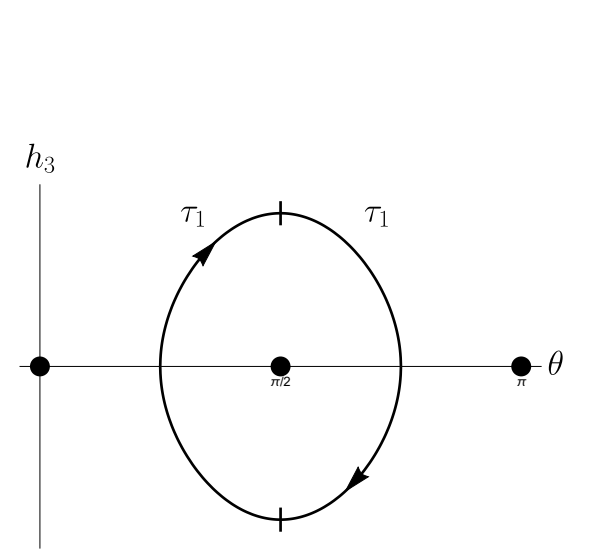

Figure 19: $\left(\theta(t), h_{3}(t)\right)$ : Case 2), domain $C_{2}$

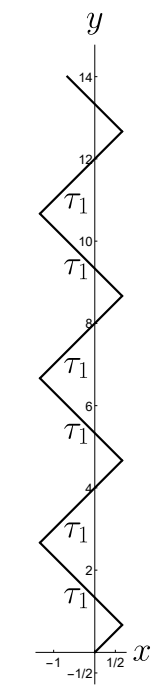

Figure 20: $(x(t), y(t))$ : Case 2), domain $C_{2}$

The control has the form

$$
\begin{array}{cccccc}
\left(u_{1}, u_{2}\right): & \ldots & (+,+) & (-,+) & (+,+) & \ldots \\
& & \tau_{1} & \tau_{1} &
\end{array}
$$

with

$$
\tau_{1}=\frac{2 \sqrt{2\left(E+h_{4}\right)}}{h_{4}} .
$$

\subsubsection{Case 2), level line $C_{3}$}

We have $h_{4}>h_{5}=0, E=0$, the corresponding trajectory is shown in Figs. 21,22 .

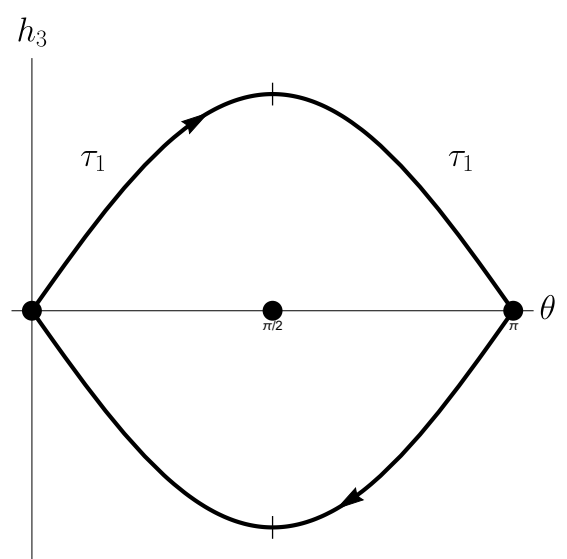

Figure 21: $\left(\theta(t), h_{3}(t)\right)$ : Case 2), level line $C_{3}$

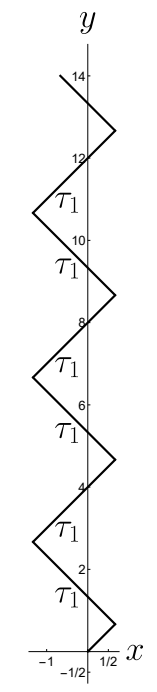

Figure 22: $(x(t), y(t))$ : Case 2), level line $C_{3}$

The control has the form 


$$
\begin{array}{cccccc}
\left(u_{1}, u_{2}\right): & \ldots & (+,+) & (-,+) & (+,+) & \ldots \\
& \tau_{1} & \tau_{1} & \tau_{1} &
\end{array}
$$

with

$$
\tau_{1}=2 \sqrt{\frac{2}{h_{4}}} .
$$

\subsubsection{Case 2), domain $C_{4}$}

We have $h_{4}>h_{5}=0, E \in\left(0, h_{4}\right)$, the corresponding trajectory is shown in Figs. 23,24 ,

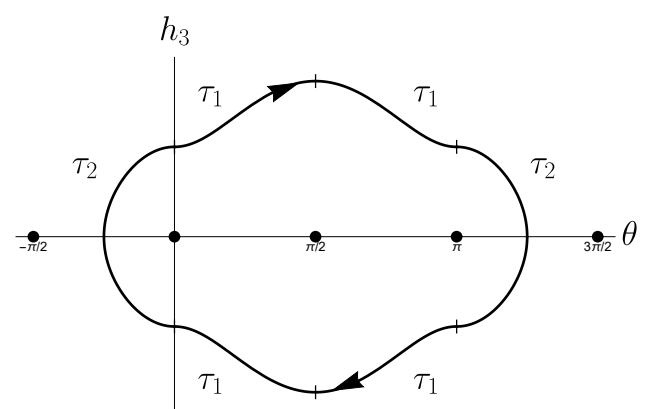

Figure 23: $\left(\theta(t), h_{3}(t)\right)$ : Case 2), domain $C_{4}$

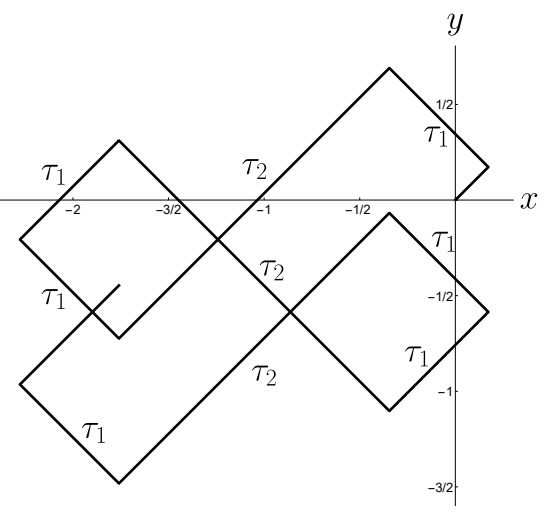

Figure 24: $(x(t), y(t))$ : Case 2), domain $C_{4}$

The control has the form

$$
\begin{array}{cccccccccc}
\left(u_{1}, u_{2}\right): & \ldots & (+,+) & (-,+) & (-,-) & (-,+) & (+,+) & (+,-) & (+,+) & \ldots \\
& \tau_{1} & \tau_{1} & \tau_{2} & \tau_{1} & \tau_{1} & \tau_{2} & \tau_{1} &
\end{array}
$$

with

$$
\tau_{1}=\frac{\sqrt{2\left(E+h_{4}\right)}-\sqrt{2 E}}{h_{4}}=\frac{2}{\sqrt{2\left(E+h_{4}\right)}+\sqrt{2 E}}, \quad \tau_{2}=\frac{2 \sqrt{2 E}}{h_{4}} .
$$

\subsubsection{Case 2), level line $C_{5}$}

We have $h_{4}>h_{5}=0, E=h_{4}$, the corresponding level line is shown in Fig. 25. 


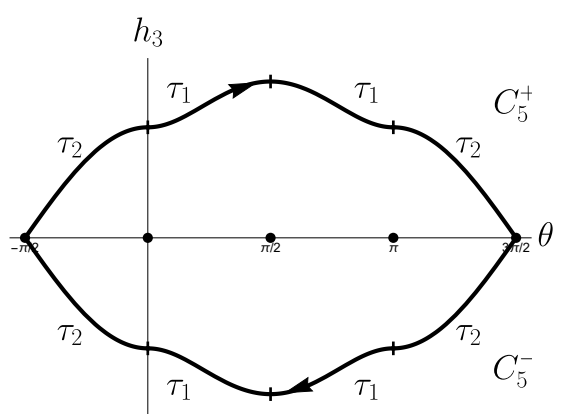

Figure 25: Case 2), level line $C_{5}$

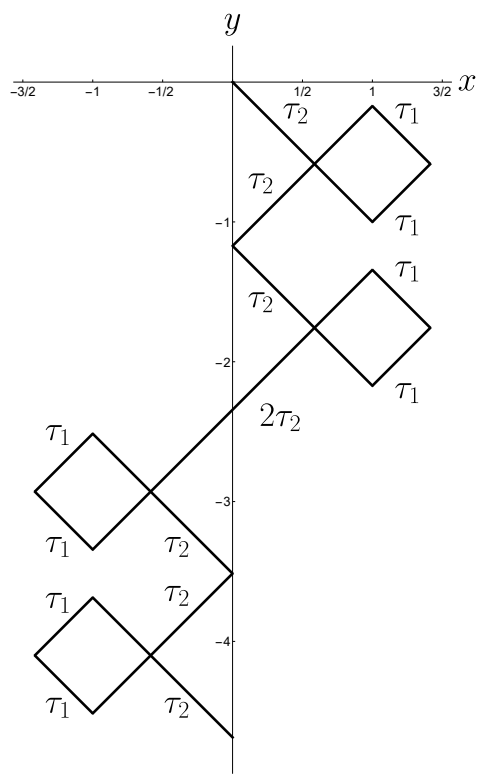

Figure 26: $(x(t), y(t))$ : Case 2), level line $C_{5}$

There are decompositions

$$
C_{5}=C_{5}^{+} \cup C_{5}^{-}, \quad C_{5}^{+}=C_{5} \cap\left\{h_{3} \geq 0\right\}, \quad C_{5}^{-}=C_{5} \cap\left\{h_{3} \leq 0\right\} .
$$

The curves $C_{5}^{+}, C_{5}^{-}$are homeomorphic to $S^{1}$, with the only singularity - a corner point $\left(\theta, h_{3}\right)=\left(\frac{3 \pi}{2}, 0\right)$.

Bang-bang controls are obtained by choosing a finite segment of the following periodic graph:

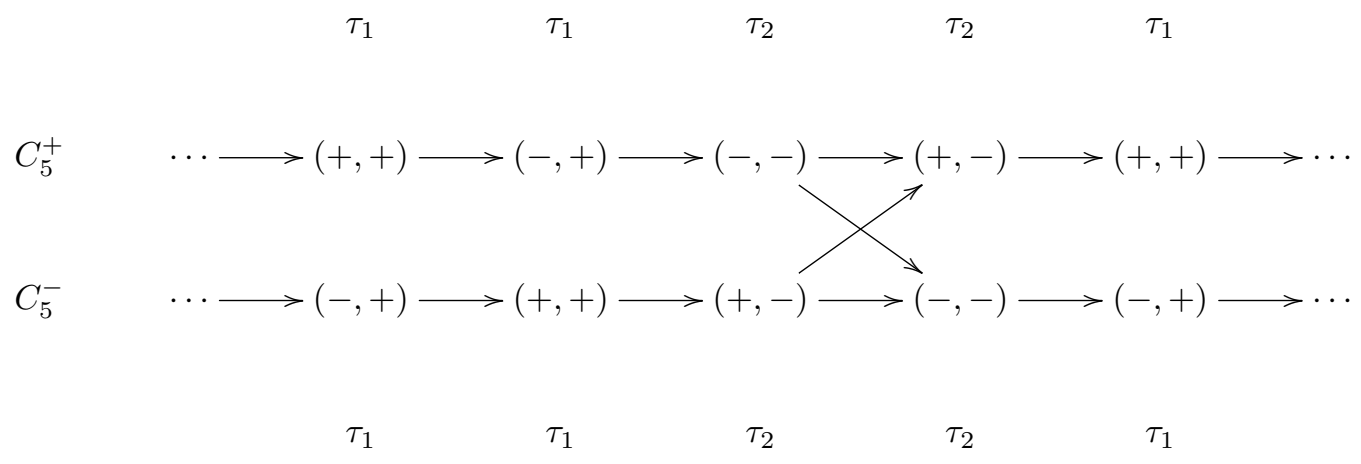

We have

$$
\tau_{1}=\frac{2-\sqrt{2}}{\sqrt{h_{4}}}, \quad \tau_{2}=\sqrt{\frac{2}{h_{4}}} .
$$

The curves $(x(t), y(t))$ corresponding to the curves $C_{5}^{+}$and $C_{5}^{-}$are shown in Figs. 27 and 28 respectively. An example of curve $(x(t), y(t))$ corresponding to two curves $C_{5}^{+}$and two curves $C_{5}^{-}$is given in Fig. 26 . 


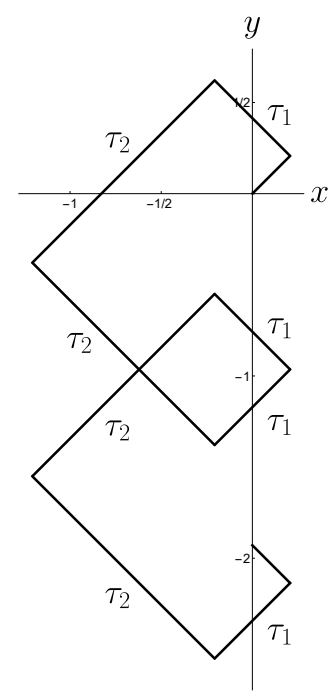

Figure 27: $(x(t), y(t))$ : Case 2), level line $C_{5}^{+}$

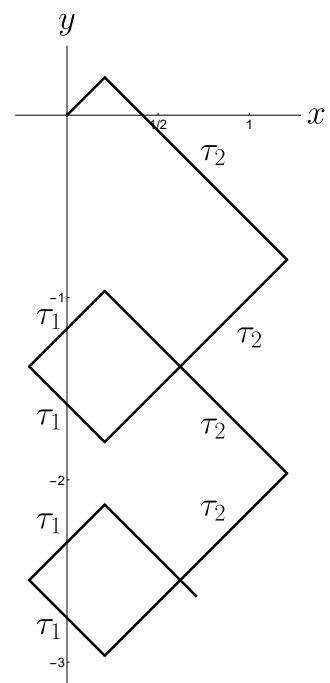

Figure 28: $(x(t), y(t))$ : Case 2), level line $C_{5}^{-}$

\subsubsection{Case 2), domain $C_{6}$}

We have $h_{4}>h_{5}=0, E>h_{4}$, the corresponding trajectory is shown in Figs. $29,30$.

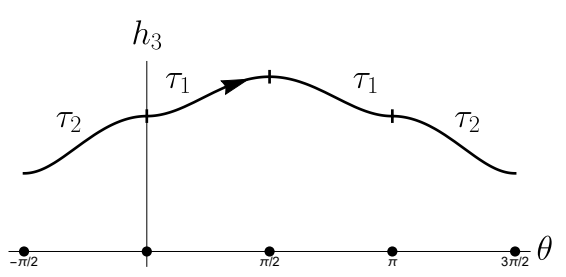

Figure 29: $\left(\theta(t), h_{3}(t)\right)$ : Case 2), domain $C_{6}$

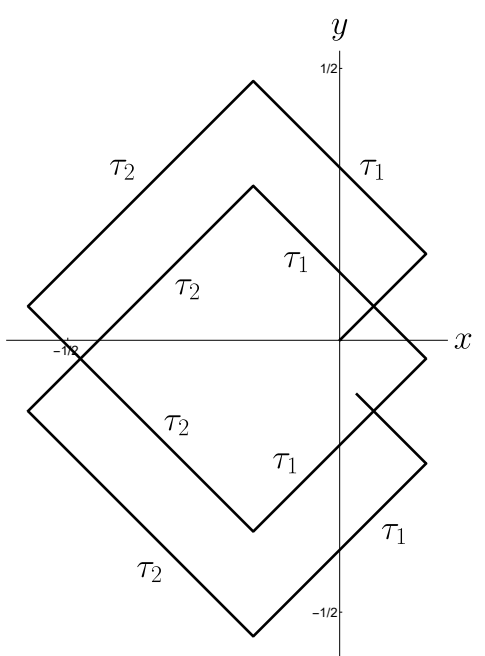

Figure 30: $(x(t), y(t))$ : Case 2), domain $C_{6}$

If $h_{3}>0$, then the control is given by

$$
\begin{array}{lllll}
\tau_{1} & \tau_{1} & \tau_{2} & \tau_{2} & \tau_{1}
\end{array}
$$

If $h_{3}<0$, then the order of switchings is opposite. We have

$$
\begin{gathered}
\tau_{1}=\frac{\sqrt{2\left(E+h_{4}\right)}-\sqrt{2 E}}{h_{4}}=\frac{2}{\sqrt{2\left(E+h_{4}\right)}+\sqrt{2 E}}, \\
\tau_{2}=\frac{\sqrt{2 E}-\sqrt{2\left(E-h_{4}\right)}}{h_{4}}=\frac{2}{\sqrt{2 E}+\sqrt{2\left(E-h_{4}\right)}} .
\end{gathered}
$$




\subsection{Case 3)}

Let $h_{4}=h_{5}>0$. Then system (2.5) has the phase portrait given in Fig. 31] see Subsubsec. 7.2.3 [17].

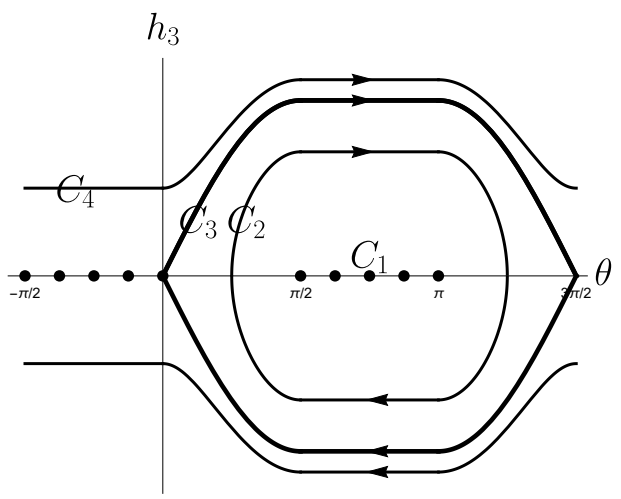

Figure 31: Phase portrait of system (2.5) in case 3 )

The domain $\left\{\lambda \in C \mid h_{4}=h_{5}>0\right\}$ of the cylinder $C=L^{*} \cap\{H=1\}$ admits a decomposition defined by the energy integral $E$ :

$$
\begin{aligned}
& \left\{\lambda \in C \mid h_{4}=h_{5}>0\right\}=\cup_{i=1}^{4} C_{i}, \\
& C_{1}=E^{-1}\left(-h_{4}\right), \quad C_{2}=E^{-1}\left(-h_{4}, 0\right), \quad C_{3}=E^{-1}\left(h_{4}\right), \quad C_{4}=E^{-1}\left(h_{4},+\infty\right) .
\end{aligned}
$$

\subsubsection{Case 3), level set $C_{1}$}

Let $h_{4}=h_{5}>0, E=-h_{4}$. Then $\theta \equiv$ const $\in[\pi / 2, \pi], h_{3} \equiv 0$.

If $\theta \in(\pi / 2, \pi)$, then $(x(t), y(t))=(-t, t)$. And if $\theta=\pi / 2(\theta=\pi)$, we get an $h_{1}$-singular (resp. $h_{2}$-singular) trajectory.

\subsubsection{Case 3), domain $C_{2}$}

We have $h_{4}=h_{5}>0, E \in\left(-h_{4}, h_{4}\right)$, the corresponding trajectory is shown in Figs. 32 , 33 .
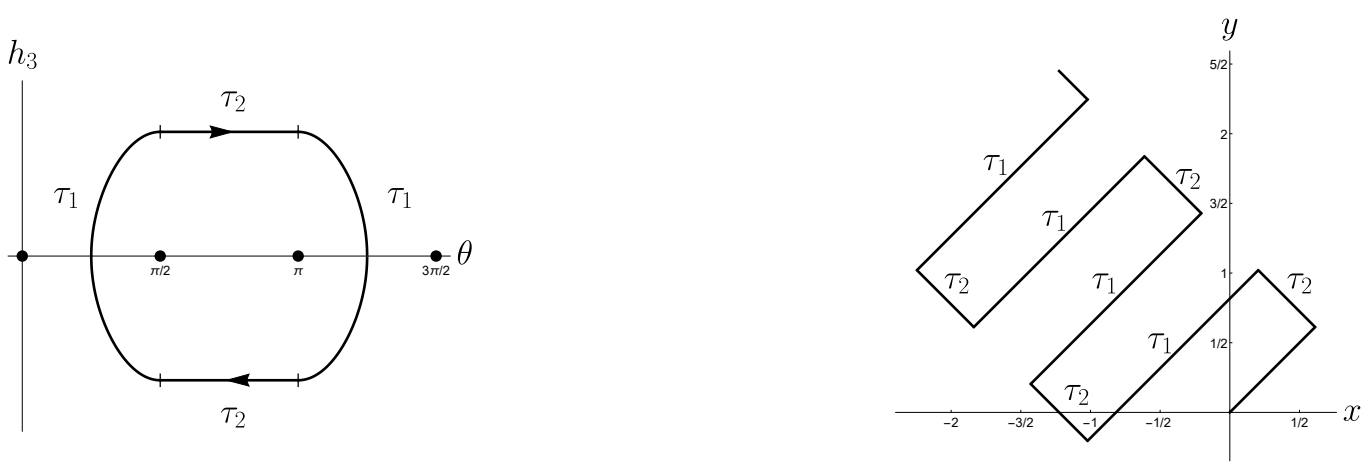

Figure 32: $\left(\theta(t), h_{3}(t)\right)$ : Case 3$)$, domain $C_{2}$

Figure 33: $(x(t), y(t))$ : Case 3), domain $C_{2}$

Then the control has the form

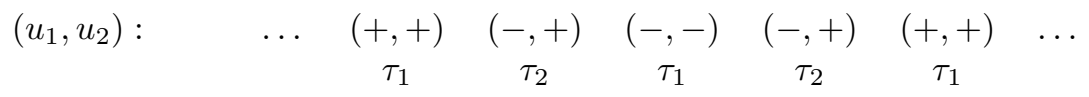


with

$$
\tau_{1}=\frac{\sqrt{2\left(E+h_{4}\right)}}{h_{4}}, \quad \tau_{2}=\frac{1}{\sqrt{2\left(E+h_{4}\right)}} .
$$

\subsubsection{Case 3 ), level line $C_{3}$}

We have $h_{4}=h_{5}>0, E=h_{4}$, the corresponding trajectory is shown in Figs. 34 35.
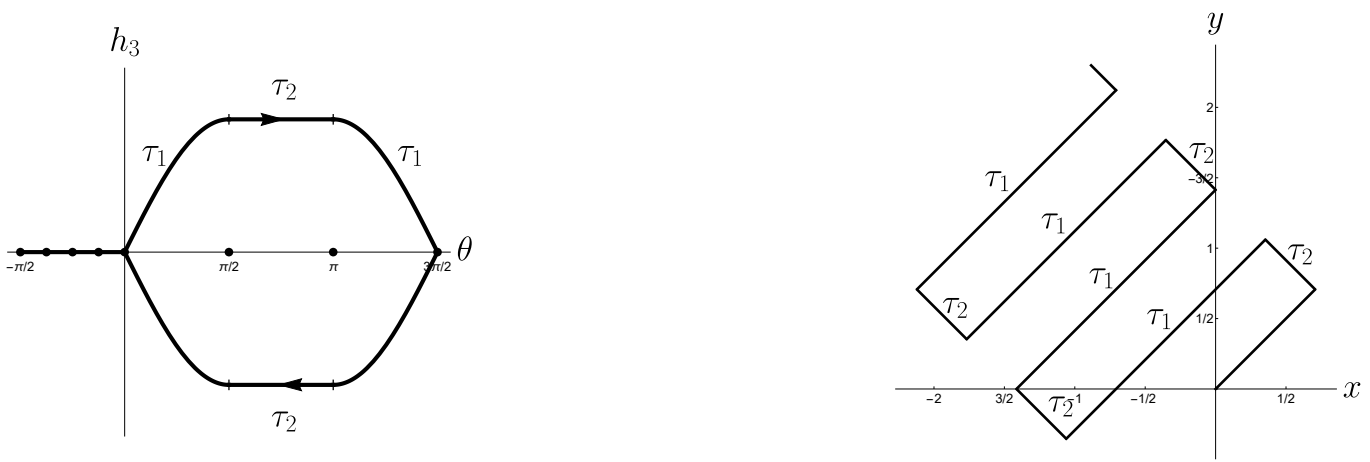

Figure 34: $\left(\theta(t), h_{3}(t)\right)$ : Case 3), level line $C_{3}$

Figure 35: $(x(t), y(t))$ : Case 3), level line $C_{3}$

The level line $C_{3}$ consists of the segment $\left\{\theta \in\left[-\frac{\pi}{2}, 0\right], h_{3}=0\right\}$ and the curve $\left\{\left(\theta, h_{3}\right) \mid h_{3}= \pm 2 \sqrt{h_{4}} \sin \theta, \theta \in\right.$ $\left.\left[0, \frac{3 \pi}{2}\right]\right\}$ homeomorphic to $S^{1}$, with two singularities - corner points $\left(\theta, h_{3}\right)=(0,0)$ and $\left(\theta, h_{3}\right)=\left(\frac{3 \pi}{2}, 0\right)$.

The bang-bang control is given by:

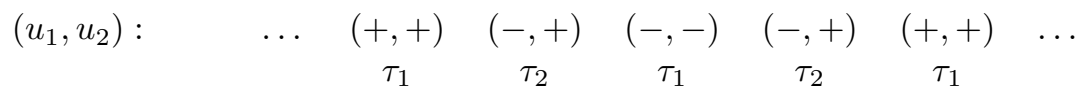

with

$$
\tau_{1}=\frac{2}{\sqrt{h_{4}}}, \quad \tau_{2}=\frac{1}{2 \sqrt{h_{4}}}
$$

\subsubsection{Case 3), domain $C_{4}$}

We have $h_{4}=h_{5}>0, E>h_{4}$, the corresponding trajectory is shown in Figs. 36, 37.

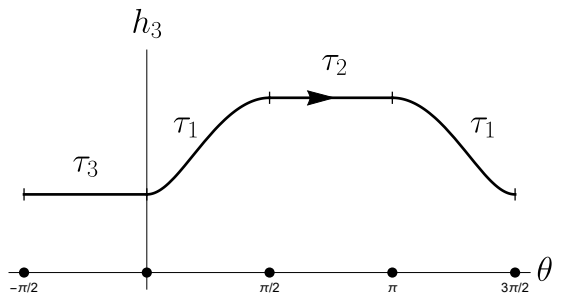

Figure 36: $\left(\theta(t), h_{3}(t)\right)$ : Case 3$)$, domain $C_{4}$

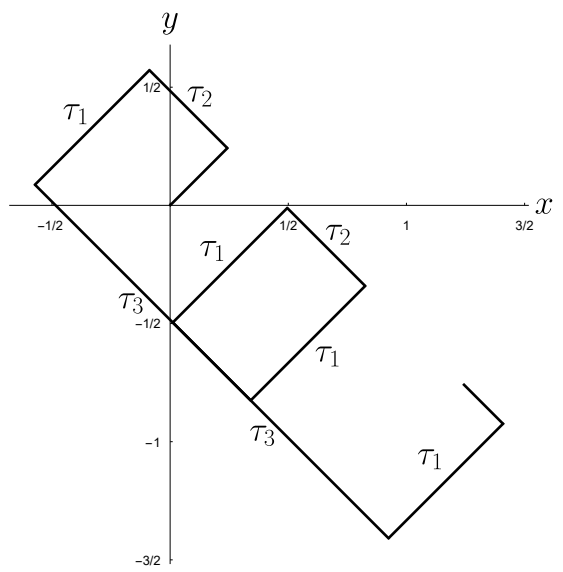

Figure 37: $(x(t), y(t))$ : Case 3), domain $C_{4}$

If $h_{3}>0$, then the control is given by: 


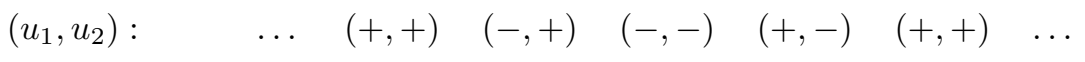

If $h_{3}<0$, then the order of switchings is opposite. We have

$$
\begin{gathered}
\tau_{1}=\frac{\sqrt{2\left(E+h_{4}\right)}-\sqrt{2\left(E-h_{4}\right)}}{2 h_{4}}=\frac{2}{\sqrt{2\left(E+h_{4}\right)}+\sqrt{2\left(E-h_{4}\right)}} \\
\tau_{2}=\frac{1}{\sqrt{2\left(E+h_{4}\right)}}, \quad \tau_{3}=\frac{1}{\sqrt{2\left(E-h_{4}\right)}} .
\end{gathered}
$$

\subsection{Case 4)}

Let $h_{4}=h_{5}=0$. Then system 2.5 has the phase portrait given in Fig. 38, see Subsubsec. 7.2 .4 [17].

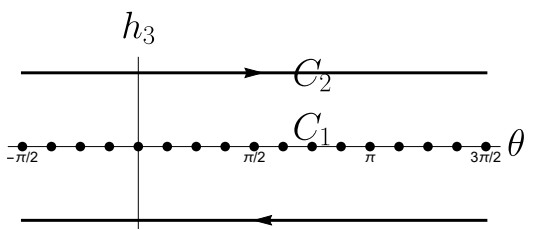

Figure 38: Phase portrait of system $(2.5)$ in case 4$)$

The domain $\left\{\lambda \in C \mid h_{4}=h_{5}=0\right\}$ of the cylinder $C=L^{*} \cap\{H=1\}$ admits a decomposition defined by the energy integral $E$ :

$$
\begin{aligned}
& \left\{\lambda \in C \mid h_{4}=h_{5}=0\right\}=C_{1} \cup C_{2}, \\
& C_{1}=E^{-1}(0), \quad C_{2}=E^{-1}(0,+\infty) .
\end{aligned}
$$

\subsubsection{Case 4), level set $C_{1}$}

We have $h_{4}=h_{5}=0, E=0$. The level set $C_{1}$ consists of fixed points $\left(\theta, h_{3}\right), h_{3}=0, \theta \neq \frac{\pi n}{2}$ that correspond to bang trajectories (which are simultaneously abnormal), and fixed points $\left(\theta, h_{3}\right)=\left(\frac{\pi n}{2}, 0\right)$ that correspond to singular trajectories (which are simultaneously abnormal as well).

\subsubsection{Case 4), domain $C_{2}$}

We have $h_{4}=h_{5}=0, E>0$, the corresponding trajectory is shown in Figs. 39,40 .

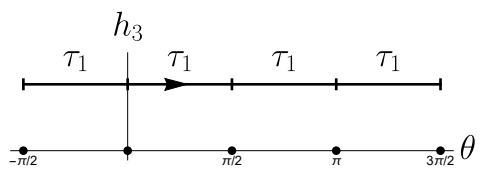

Figure 39: $\left(\theta(t), h_{3}(t)\right)$ : Case 4$)$, domain $C_{2}$

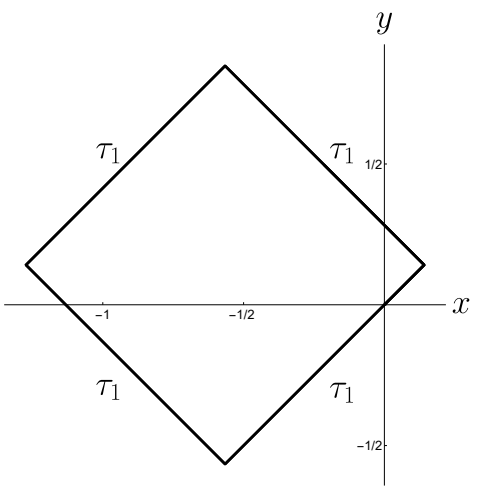

Figure 40: $(x(t), y(t))$ : Case 4), domain $C_{2}$

If $h_{3}>0$, then the control $u$ is given by: 


$$
\begin{array}{cccccccc}
\left(u_{1}, u_{2}\right): & \ldots & (+,+) & (-,+) & (-,-) & (+,-) & (+,+) & \ldots \\
& \tau_{1} & \tau_{1} & \tau_{1} & \tau_{1} & \tau_{1} &
\end{array}
$$

If $h_{3}<0$, then the order of switchings is opposite. We have

$$
\tau_{1}=\frac{1}{\sqrt{2 E}}
$$

On the basis of the results obtained in this section we obtain the following statement.

Corollary 1. For all bang-bang trajectories, duration of bang arcs is a function of Casimirs: $\tau_{i}=\tau_{i}\left(h_{4}, h_{5}, E\right)$, except the first and the last arcs.

\section{Optimality of bang-bang trajectories}

In this section we obtain upper bounds on the number of switchings on bang-bang minimizers.

\subsection{Bang-bang trajectories with low energy $E$}

Theorem 2. If a bang-bang extremal $\lambda_{t}, t \in[0,+\infty)$, satisfies the inequality

$$
\min \left(-\left|h_{4}\right|,-\left|h_{5}\right|\right)<E \leq \max \left(-\left|h_{4}\right|,-\left|h_{5}\right|\right)
$$

then it is optimal, i.e., $t_{\text {cut }}\left(\lambda_{0}\right)=+\infty$.

Proof. All three functions $\min \left(-\left|h_{4}\right|,-\left|h_{5}\right|\right), E, \max \left(-\left|h_{4}\right|,-\left|h_{5}\right|\right)$ are invariant w.r.t. the group of symmetries of the square $\left\{\left|h_{1}\right|+\left|h_{2}\right|=1\right\}$ (see Sec. 7.1 [17]), thus it suffices to prove this theorem for the fundamental domain of this group $\left\{h_{4} \geq h_{5} \geq 0\right\}$. On this domain inequality 4.1 turns into the inequality $-h_{4}<E \leq-h_{5} \leq 0$, which is equivalent to the union of the following inequalities:

(a) $-h_{4}<E \leq-h_{5} \leq 0$,

(b) $-h_{4}<E \leq-h_{5}=0$.

Case (a) is exactly Case 1), domain $C_{2}$ (Subsec. 3.1.2 and Case 1), level line $C_{3}$ (Subsec. 3.1.3). And case (b) is exactly Case 2), domain $C_{2}$ (Subsec. 3.2.2) and Case 2), level line $C_{3}$ (Subsec. 3.2.3). In all these cases $\theta(t) \in[0, \pi]$, and $\theta(t)$ takes extreme values $0, \pi$ at isolated instants of time $t$. Thus $h_{2}\left(\lambda_{t}\right)>0$ for almost all $t$, whence $u_{2}(t) \equiv 1$ for almost all $t$. By Lemma 2 [17, the control $u(t)$ is optimal.

The domain in the phase cylinder of system 2.5 corresponding to inequalities 4.1) is shown in Fig. 41.

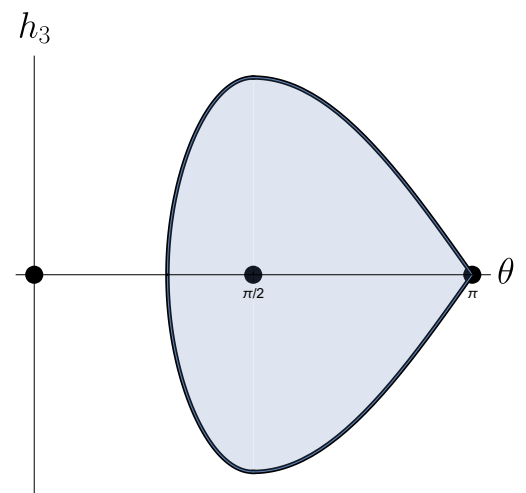

Figure 41: Domain 4.1 
Remark. It is easy to see that under condition (4.1) a bang-bang trajectory is simultaneously a singular trajectory, i.e., $q(t)=\pi\left(\tilde{\lambda}_{t}\right)$, while $\tilde{\lambda}_{t}$ is an $h_{1}$-singular extremal linearly independent of $\lambda_{t}$. One can show that there exists also a bang-bang extremal $\bar{\lambda}_{t}$, linearly independent of $\lambda_{t}$ and $\tilde{\lambda}_{t}$, such that $q(t)=\pi\left(\bar{\lambda}_{t}\right)$. Thus the trajectory $q(t)$ is a projection of at least three linearly independent extremals (in this case the extremal trajectory is said to have corank not less than 3 [12]). Thus the below necessary optimality condition (Th. 3) is not applicable to a trajectory $q(t)$ under condition 4.1 .

\subsection{Bound of the number of switchings on bang-bang trajectories with high energy $E$}

\subsubsection{Some necessary results}

We obtain an upper bound on the number of switchings on optimal bang-bang trajectories via the following theorem due to A. Agrachev and R. Gamkrelidze.

Theorem $3($ 14, 15]). Let $(q(\cdot), u(\cdot))$ be an extremal pair for problem (2.1)-(2.3) and let $\lambda$. be an extremal lift of $q(\cdot)$. Assume that $\lambda$. is the unique extremal lift of $q(\cdot)$, up to multiplication by a positive scalar. Assume that there exist $0=t_{0}<t_{1}<t_{2}<\cdots<t_{k}<\tau_{k+1}=T$ and $u^{0}, \ldots, u^{k} \in U$ such that $u(\cdot)$ is constantly equal to $u^{j}$ on $\left(\tau_{j}, \tau_{j+1}\right)$ for $j=0, \ldots, k$.

Fix $j=1, \ldots, k$. For $i=0, \ldots, k$ let $Y_{i}=u_{1}^{i} X_{1}+u_{2}^{i} X_{2}$ and define recursively the operators

$$
\begin{aligned}
& P_{j}=P_{j-1}=\operatorname{Id}_{\operatorname{Vec}(M)}, \\
& P_{i}=P_{i-1} \circ e^{\left(t_{i}-t_{i-1}\right) \text { ad } Y_{i-1}}, \quad i=j+1, \ldots, k, \\
& P_{i}=P_{i+1} \circ e^{-\left(t_{i+2}-t_{i+1}\right) \text { ad } Y_{i+1}}, \quad i=0, \ldots, j-2 .
\end{aligned}
$$

Define the vector fields

$$
Z_{i}=P_{i}\left(Y_{i}\right), \quad i=0, \ldots, k
$$

Let $Q$ be the quadratic form

$$
Q(\alpha)=\sum_{0 \leq i<l \leq k} \alpha_{i} \alpha_{l}\left\langle\lambda_{t_{j}},\left[Z_{i}, Z_{l}\right]\left(q\left(t_{j}\right)\right)\right\rangle
$$

defined on the space

$$
W=\left\{\alpha=\left(\alpha_{0}, \ldots, \alpha_{k}\right) \in \mathbb{R}^{k+1} \mid \sum_{i=0}^{k} \alpha_{i}=0, \quad \sum_{i=0}^{k} \alpha_{i} Z_{i}\left(q\left(t_{j}\right)\right)=0\right\} .
$$

If $Q$ is not negative-semidefinite, then $q(\cdot)$ is not optimal.

We will check the sign of the quadratic form $\left.Q\right|_{W}$ via the following test.

Consider a quadratic form

$$
A(x)=\sum_{i, j=1}^{n} a_{i j} x_{i} x_{j}, \quad a_{i j}=a_{j i}, \quad x_{i} \in \mathbb{R} .
$$

Denote a minor

$$
A\left(\begin{array}{cccc}
i_{1} & i_{2} & \ldots & i_{p} \\
i_{1} & i_{2} & \ldots & i_{p}
\end{array}\right)=\left|\begin{array}{rrrr}
a_{i_{1} i_{1}} & a_{i_{1} i_{2}} & \ldots & a_{i_{1} i_{p}} \\
a_{i_{2} i_{1}} & a_{i_{2} i_{2}} & \ldots & a_{i_{2} i_{p}} \\
\ldots \ldots & \ldots & \ldots & \ldots \\
\ldots & \ldots & \ldots \\
a_{i_{p} i_{1}} & a_{i_{p} i_{2}} & \ldots & a_{i_{p} i_{p}}
\end{array}\right|
$$

Theorem 4 ([16]). A quadratic form $A(x)$ is negative-semidefinite iff the following inequalities hold:

$$
(-1)^{p} A\left(\begin{array}{cccc}
i_{1} & i_{2} & \ldots & i_{p} \\
i_{1} & i_{2} & \ldots & i_{p}
\end{array}\right) \geq 0, \quad 1 \leq i_{1}<i_{2}<\cdots<i_{p} \leq n, \quad p=1,2, \ldots, n .
$$




\subsubsection{Case 1), domain $C_{4}$}

Theorem 5. Let $h_{4}>h_{5}>0, E \in\left(-h_{5}, h_{5}\right)$. Then any bang-bang control with 8 switchings is not optimal.

Proof. Consider a control starting from $(1,-1)$ and having $k=8$ switchings (controls starting from other values are considered similarly). We apply Th. 3 and show that such control is not optimal. We have $0=t_{0}<t_{1}<\cdots<$ $t_{9}=T$, where

$$
\begin{aligned}
& t_{1} \in\left(0, \tau_{1}\right], \quad t_{2}-t_{1}=t_{4}-t_{3}=t_{6}-t_{5}=t_{8}-t_{7}=\tau_{2}, \\
& t_{3}-t_{2}=t_{7}-t_{6}=\tau_{3}, \quad t_{5}-t_{4}=\tau_{1}, \quad t_{9}-t_{8} \in\left(0, \tau_{1}\right],
\end{aligned}
$$

and the values $\tau_{1}, \tau_{2}, \tau_{3}$ are defined in Subsec. 3.1 .4 Further, we have

$$
\begin{aligned}
& \left.u\right|_{\left(t_{0}, t_{1}\right)}=\left.u\right|_{\left(t_{4}, t_{5}\right)}=\left.u\right|_{\left(t_{8}, t_{9}\right)}=(1,1), \\
& \left.u\right|_{\left(t_{1}, t_{2}\right)}=\left.u\right|_{\left(t_{3}, t_{4}\right)}=\left.u\right|_{\left(t_{5}, t_{6}\right)}=\left.u\right|_{\left(t_{7}, t_{8}\right)}=(-1,1), \\
& \left.u\right|_{\left(t_{2}, t_{3}\right)}=\left.u\right|_{\left(t_{6}, t_{7}\right)}=(-1,-1),
\end{aligned}
$$

see Fig. 6. We apply Th. 3 in the case $k=8, j=1$. We use the basis $\left(X_{+}, X_{-}, X_{3}, X_{++}, X_{--}\right)$in the Lie algebra $L$, where $X_{+}=X_{1}+X_{2}, X_{-}=X_{1}-X_{2}, X_{++}=X_{4}+X_{5}, X_{--}=X_{4}-X_{5}$. Then

$$
Y_{0}=-Y_{2}=Y_{4}=-Y_{6}=Y_{8}=X_{+}, \quad Y_{1}=Y_{3}=Y_{5}=Y_{7}=-X_{-} .
$$

Further,

$$
\begin{array}{ll}
P_{1}=P_{0}=\mathrm{Id}, & P_{5}=P_{4} \circ e^{\tau_{1} \text { ad } X_{+},}, \\
P_{2}=e^{-\tau_{2} \text { ad } X_{-},} & P_{6}=P_{5} \circ e^{-\tau_{2} \text { ad } X_{-}}, \\
P_{3}=P_{2} \circ e^{-\tau_{3} \text { ad } X_{+},} & P_{7}=P_{6} \circ e^{-\tau_{3} \text { ad } X_{+}}, \\
P_{4}=P_{3} \circ e^{-\tau_{2} \text { ad } X_{-}}, & P_{8}=P_{7} \circ e^{-\tau_{2} \text { ad } X_{-}} .
\end{array}
$$

Thus

$$
\begin{aligned}
& Z_{0}=X_{+} \\
& Z_{1}=-X_{-} \\
& Z_{2}=-X_{+}+2 \tau_{2} X_{3}-\tau_{2}^{2} X_{--} \\
& Z_{3}=-X_{-}-2 \tau_{3} X_{3}+\tau_{3}^{2} X_{++}+2 \tau_{2} \tau_{3} X_{--} \\
& Z_{4}=X_{+}-4 \tau_{2} X_{3}+2 \tau_{2} \tau_{3} X_{++}+4 \tau_{2}^{2} X_{--} \\
& Z_{5}=-X_{-}+\left(2 \tau_{1}-2 \tau_{3}\right) X_{3}+\left(\tau_{1}^{2}-2 \tau_{1} \tau_{3}+\tau_{3}^{2}\right) X_{++}+\left(2 \tau_{2} \tau_{3}-4 \tau_{1} \tau_{2}\right) X_{--}, \\
& Z_{6}=-X_{+}+6 \tau_{2} X_{3}+\left(2 \tau_{1} \tau_{2}-4 \tau_{2} \tau_{3}\right) X_{++}-9 \tau_{2}^{2} X_{--} \\
& Z_{7}=-X_{-}+\left(2 \tau_{1}-4 \tau_{3}\right) X_{3}+\left(4 \tau_{3}^{2}-4 \tau_{1} \tau_{3}+\tau_{1}^{2}\right) X_{++}+\left(8 \tau_{2} \tau_{3}-4 \tau_{1} \tau_{2}\right) X_{--}, \\
& Z_{8}=X_{+}-8 \tau_{2} X_{3}+\left(8 \tau_{2} \tau_{3}-4 \tau_{2} \tau_{1}\right) X_{++}+16 \tau_{2}^{2} X_{--}
\end{aligned}
$$

Introduce the notation:

$$
c=h_{3}, \quad a=h_{4}+h_{5}, \quad b=h_{4}-h_{5} .
$$


Then $Q(\alpha)=\sum_{i, l=0}^{8} \sigma_{i l} \alpha_{i} \alpha_{l}$, where

$$
\begin{array}{lll}
\sigma_{01}=2 c, & \sigma_{16}=2 c-6 \tau_{2} b, & \sigma_{37}=\left(2 \tau_{3}-2 \tau_{1}\right) b, \\
\sigma_{02}=2 \tau_{2} a, & \sigma_{17}=\left(4 \tau_{3}-2 \tau_{1}\right) b, & \sigma_{38}=-2 c+2 \tau_{3} a+8 \tau_{2} b, \\
\sigma_{03}=2 c-2 \tau_{3} a, & \sigma_{18}=-2 c+8 \tau_{2} b, & \sigma_{45}=2 c+\left(2 \tau_{1}-2 \tau_{3}\right) a-4 \tau_{2} b, \\
\sigma_{04}=-4 \tau_{2} a, & \sigma_{23}=-2 c+2 \tau_{3} a+2 \tau_{2} b, & \sigma_{46}=2 \tau_{2} a, \\
\sigma_{05}=2 c+\left(2 \tau_{1}-2 \tau_{3}\right) a, & \sigma_{24}=2 \tau_{2} a, & \sigma_{47}=2 c-4 \tau_{2} b+\left(2 \tau_{1}-4 \tau_{3}\right) a, \\
\sigma_{06}=6 \tau_{2} a, & \sigma_{25}=-2 c-\left(2 \tau_{1}-2 \tau_{3}\right) a+2 \tau_{2} b, & \sigma_{48}=-4 \tau_{2} a, \\
\sigma_{07}=2 c+\left(2 \tau_{1}-4 \tau_{3}\right) a, & \sigma_{26}=-4 \tau_{2} a, & \sigma_{56}=2 c+\left(2 \tau_{1}-2 \tau_{3}\right) a-6 \tau_{2} b, \\
\sigma_{08}=-8 \tau_{2} a, & \sigma_{27}=-2 c-\left(2 \tau_{1}-4 \tau_{3}\right) a+2 \tau_{2} b, & \sigma_{57}=2 \tau_{3} b, \\
\sigma_{12}=2 c-2 \tau_{2} b, & \sigma_{28}=6 \tau_{2} a, & \sigma_{58}=-2 c+\left(2 \tau_{3}-2 \tau_{1}\right) a+8 \tau_{2} b, \\
\sigma_{13}=2 \tau_{3} b, & \sigma_{34}=-2 c+2 \tau_{3} a+4 \tau_{2} b, & \sigma_{67}=-2 c+\left(4 \tau_{3}-2 \tau_{1}\right) a+6 \tau_{2} b, \\
\sigma_{14}=-2 c+4 \tau_{2} b, & \sigma_{35}=-2 \tau_{1} b, & \sigma_{68}=2 \tau_{2} a, \\
\sigma_{15}=\left(2 \tau_{3}-2 \tau_{1}\right) b, & \sigma_{36}=2 c-2 \tau_{3} a-6 \tau_{2} b, & \sigma_{78}=-2 c+\left(4 \tau_{3}-2 \tau_{1}\right) a+8 \tau_{2} b .
\end{array}
$$

Further,

$$
\begin{aligned}
& W=\left\{\left(\alpha_{0}, \ldots, \alpha_{8}\right) \in \mathbb{R}^{9} \mid \sum_{i=0}^{8} \alpha_{i}=0, \quad \sum_{i=0}^{8} \alpha_{i} Z_{i}\left(q\left(t_{1}\right)\right)=0\right\} \\
& =\left\{\left(\alpha_{0}, \ldots, \alpha_{8}\right) \in \mathbb{R}^{9} \quad \mid \quad \alpha_{3}=\left(4 \tau_{2} \alpha_{0}+\left(2 \tau_{3}-\tau_{1}\right) \alpha_{1}-2 \tau_{2} \alpha_{2}\right) / \tau_{1},\right. \\
& \alpha_{5}=\left(-4 \tau_{2} \alpha_{0}+\left(\tau_{1}-2 \tau_{3}\right) \alpha_{1}+2 \tau_{2} \alpha_{2}\right) / \tau_{1}-2 \tau_{2} \alpha_{4} / \tau_{3}, \\
& \left.\alpha_{6}=-\alpha_{2}, \quad \alpha_{7}=-\alpha_{1}+2 \tau_{2} \alpha_{4} / \tau_{3}, \quad \alpha_{8}=-\alpha_{0}-\alpha_{4}\right\}, \\
& \left.Q\right|_{W}=\frac{4}{\tau_{1} \tau_{3}} \sum_{i, j=0,1,2,4} a_{i j} \alpha_{i} \alpha_{j}, \\
& a_{00}=2 \tau_{2} \tau_{3}\left(-a \tau_{1}+4 b \tau_{2}\right), \quad a_{02}=2 \tau_{2} \tau_{3}\left(a \tau_{1}-2 b \tau_{2}\right), \\
& a_{11}=b \tau_{3}^{2}\left(-\tau_{1}+2 \tau_{3}\right), \quad a_{04}=2 \tau_{2} \tau_{3}\left(-c+2 b \tau_{2}+a\left(\tau_{3}-\tau_{1}\right)\right), \\
& a_{22}=\tau_{2} \tau_{3}\left(-a \tau_{1}+2 b \tau_{2}\right), \quad a_{12}=-\tau_{3}\left(\tau_{1}-2 \tau_{3}\right)\left(a \tau_{1}-2 b \tau_{2}\right) / 2 \text {, } \\
& a_{44}=-\tau_{1} \tau_{2}\left(2 b \tau_{2}+a \tau_{3}\right), \quad a_{14}=\tau_{3}\left(2 b \tau_{2}\left(\tau_{1}+2 \tau_{3}\right)+\tau_{3}\left(-2 c-a \tau_{1}+2 a \tau_{3}\right)\right) / 2, \\
& a_{01}=4 b \tau_{2} \tau_{3}^{2}, \quad a_{24}=-\tau_{2}\left(-2 a \tau_{1}^{2}+6 b \tau_{1} \tau_{2}+a \tau_{1} \tau_{3}+4 b \tau_{2} \tau_{3}+2 a \tau_{3}^{2}-2 c\left(\tau_{1}+\tau_{3}\right)\right) / 2 \text {. }
\end{aligned}
$$

Then

$$
\begin{gathered}
A\left(\begin{array}{ll}
0 & 1 \\
0 & 1
\end{array}\right)=\left|\begin{array}{cc}
a_{00} & a_{01} \\
a_{01} & a_{11}
\end{array}\right|=\frac{512(1+Y)(X+Y)(X+Y-\sqrt{(1+Y)(X+Y)})}{h_{4}(1+X)^{4}}, \\
X=\frac{h_{5}}{h_{4}} \in(0,1), \quad Y=\frac{E}{h_{4}} \in(-X, X) .
\end{gathered}
$$

Thus $A\left(\begin{array}{ll}0 & 1 \\ 0 & 1\end{array}\right)<0$, i.e., the quadratic form $\left.Q\right|_{W}$ is not negative semidefinite. By Th. 3 the control $u$ is not optimal.

\subsubsection{Bound of the number of switchings in the general case of high energy $E$}

The rest cases are considered similarly to Th. 5 .

- Case 1), $\cup_{i=5}^{8} C_{i}$,

- Case 2), $\cup_{i=4}^{6} C_{i}$,

- Case 3),

- Case 4). 
In all these cases Th. 3 and Th. 4 imply that $k=12$ switchings are not optimal.

Passing from the fundamental domain $\left\{h_{4} \geq h_{5} \geq 0\right\}$ of the group $G$ to the whole plane $\left(h_{4}, h_{5}\right)$, we get the following general bound of the number of switchings.

Theorem 6. If $E>\max \left(-\left|h_{4}\right|,-\left|h_{5}\right|\right)$, then optimal bang-bang trajectories have no more than 11 switchings.

In particular, in this case $t_{\text {cut }}(\lambda)<+\infty$.

\section{General form of normal extremals}

Now we prove that the list of types of normal extremals given in Sec. 2 is complete.

Theorem 7. If $\lambda_{t}, t \in[0, T]$, is a normal extremal, then there exist $0 \leq t_{1}<t_{2}<\cdots<t_{n} \leq T$, for which the following conditions hold:

- $h_{1} h_{2}\left(\lambda_{t_{i}}\right)=0, \quad i=1, \ldots, n$,

- for any $i=1, \ldots, n-1$, one of the following conditions is satisfied:

$$
\begin{array}{ll}
\left.h_{1} h_{2}\left(\lambda_{t}\right)\right|_{\left(t_{i}, t_{i+1}\right)} \neq 0 \text { or } \\
\left.h_{1}\left(\lambda_{t}\right)\right|_{\left[t_{i}, t_{i+1}\right]} \equiv 0,\left.\quad h_{2}\left(\lambda_{t}\right)\right|_{\left(_{i}, t_{i+1}\right)} \neq 0 \text { or } \\
\left.h_{2}\left(\lambda_{t}\right)\right|_{\left[t_{i}, t_{i+1}\right]} \equiv 0,\left.\quad h_{1}\left(\lambda_{t}\right)\right|_{\left(t_{i}, t_{i+1}\right)} \neq 0 .
\end{array}
$$

Proof. Introduce the sets

$$
Z=\left\{t \in[0, T] \mid h_{1} h_{2}\left(\lambda_{t}\right)=0\right\}, \quad N=[0, T] \backslash Z .
$$

The set $N$ is open in $[0, T]$, thus it consists of a finite or countable number of open intervals (and, may be, two half-open intervals near the endpoints of $[0, T]$ ). We prove that $N$ consists of a finite number of intervals. By contradiction, suppose that $N$ is a countable union of non-intersecting intervals. Choosing a point in each interval, construct a sequence $\left\{\tau_{n} \mid n \in \mathbb{N}\right\} \subset N$. Passing to a subsequence, we can assume that $\exists \lim _{n \rightarrow \infty} \tau_{n}=\bar{t} \in[0, T]$, and $\tau_{n}<\bar{t}$ for all $n \in \mathbb{N}$ (or $\tau_{n}>\bar{t}$ for all $n \in \mathbb{N}$, which is considered similarly). Since the points $\tau_{n}$ belong to different connected components of $N$, there exists a sequence $\left\{s_{n}\right\}, n \in \mathbb{N}$, such that $s_{n} \in Z, \quad \tau_{n}<s_{n}<\tau_{n+1}<$ $s_{n+1}<\cdots<\bar{t}, \quad n \in \mathbb{N}$. Thus $\lim _{n \rightarrow \infty} s_{n}=\bar{t}$. Since $h_{1} h_{2}\left(\lambda_{\tau_{n}}\right) \neq 0$, there exists an interval $\left(\alpha_{n}, \beta_{n}\right) \ni \tau_{n}$ such that $\left.h_{1} h_{2}\left(\lambda_{t}\right)\right|_{\left(\alpha_{n}, \beta_{n}\right)} \neq 0, n \in \mathbb{N}$. Each extremal arc $\left.\lambda_{t}\right|_{\left(\alpha_{n}, \beta_{n}\right)}, n \in \mathbb{N}$, is a bang arc, and the Casimirs $h_{4}, h_{5}, E$ take the same value on each of these arcs. Thus duration of all bang arcs is separated from zero: $\beta_{n}-\alpha_{n} \geq C>0$, $C=C\left(h_{4}, h_{5}, E\right)$, see Cor. 1. Then $s_{n-1}-s_{n} \geq \beta_{n}-\alpha_{n} \geq C>0$, which contradicts the equality $\lim _{n \rightarrow \infty} s_{n}=\bar{t}$. Thus $N$ consists of a finite number of intervals.

Let $\left.h_{1} h_{2}\left(\lambda_{t}\right)\right|_{\left[t_{i}, t_{i+1}\right]} \equiv 0$. Notice that if $h_{j}\left(\lambda_{t}\right)=0$, then $h_{3-j}\left(\lambda_{t}\right) \neq 0, j=1,2$, since $H\left(\lambda_{t}\right)=\left(\left|h_{1}\right|+\left|h_{2}\right|\right)\left(\lambda_{t}\right)>0$ for a normal extremal $\lambda_{t}$. Take any $\bar{t} \in\left[t_{i}, t_{i+1}\right]$, then we can assume that $h_{1}\left(\lambda_{\bar{t}}\right)=0, h_{2}\left(\lambda_{\bar{t}}\right) \neq 0$ (the case $h_{2}\left(\lambda_{\bar{t}}\right)=$ $0, h_{1}\left(\lambda_{\bar{t}}\right) \neq 0$ is considered similarly). Then there exists a neighborhood $O(\bar{t}) \subset\left[t_{1}, t_{2}\right]$ such that $\left.h_{2}\left(\lambda_{t}\right)\right|_{O(\bar{t})} \neq 0$, thus $\left.h_{1}\left(\lambda_{t}\right)\right|_{O(\bar{t})} \equiv 0$. Thus the set $\left\{t \in\left[t_{i}, t_{i+1}\right] \mid h_{1}\left(\lambda_{t}\right)=0\right\}$ is open and closed in $\left[t_{i}, t_{i+1}\right]$, so it coincides with $\left[t_{i}, t_{i+1}\right]$. In other words,

$$
\left.h_{1}\left(\lambda_{t}\right)\right|_{\left[t_{i}, t_{i+1}\right]} \equiv 0,\left.\quad h_{2}\left(\lambda_{t}\right)\right|_{\left(t_{i}, t_{i+1}\right)} \neq 0 .
$$

Summing up, any normal extremal is either bang-bang, or singular, or mixed.

\section{$6 \quad$ Mixed extremals}

Consider an extremal $\lambda_{t}, t \in[0, T]$, and let $0 \leq \alpha<\beta<\gamma \leq T$. Let the arc $\left.\lambda_{t}\right|_{[\alpha, \beta]}$ be bang-bang, and let the arc $\left.\lambda_{t}\right|_{[\beta, \gamma]}$ be singular. Then we say that the bang-bang $\left.\operatorname{arc} \lambda_{t}\right|_{[\alpha, \beta]}$ adjoins the singular $\left.\operatorname{arc} \lambda_{t}\right|_{[\beta, \gamma]}$ at the point $\lambda_{\beta}$. Similarly in the case when a singular arc precedes a bang-bang arc.

Notice that singular arcs of types (a), (b) were described in Theorems 3,4 [17].

Proposition 2. Let $h_{4} \geq h_{5} \geq 0$.

A singular arc can adjoin a bang-bang arc only at points $\lambda_{\bar{t}}$ that satisfy the following conditions:

- $\theta=\frac{3 \pi}{2}, h_{3}=0,0 \leq h_{5} \leq h_{4}$ ( $h_{1}$-singular arc of type (b) adjoins a bang-bang arc), 
- $\theta=0, h_{3}=0,0<h_{5}=h_{4}$

( $h_{2}$-singular arc of type (b) adjoins a bang-bang arc).

Proof. Singular arcs of type (a) cannot adjoin bang-bang arcs since these singular arcs satisfy the equalities $h_{3}=$ $h_{4}=h_{5}=0$ (see Th. 3, 4 [17]), but these equalities cannot hold on bang-bang extremals (see Subsec. 3.4 ).

$h_{1}$-singular arcs of type (b) satisfy the conditions:

$$
\theta=\frac{\pi}{2}+\pi n, \quad h_{3}=0, \quad 0<h_{5} \leq h_{4} .
$$

The point $\theta=\frac{\pi}{2}, h_{3}=0$ is an equilibrium point of the phase portrait of the reduced Hamiltonian system of PMP, thus the equality $\left(\theta, h_{3}\right)=\left(\frac{\pi}{2}, 0\right)$ cannot hold on a bang-bang extremal. Similarly, $h_{2}$-singular arc of type (b) satisfies the conditions

$$
\theta=\pi n, \quad h_{3}=0, \quad h_{4}=h_{5}=0,
$$

and the equality $\left(\theta, h_{3}\right)=(\pi, 0)$ cannot hold on a bang-bang extremal.

Notice that singular controls that adjoin bang-bang controls are constant. Thus all mixed controls are piecewise constant, and Th. 3 can be used for bounding the number of switchings on optimal mixed trajectories.

Mixed extremals are schematically shown in Figs. 42, 46. Small dashed circles near the points $\left(\theta, h_{3}\right)=\left(\frac{3 \pi}{2}, 0\right)$ and $\left(\theta, h_{3}\right)=(0,0)$ denote singular arcs that adjoin bang-bang arcs. Singular arcs are shown by dashed segments.

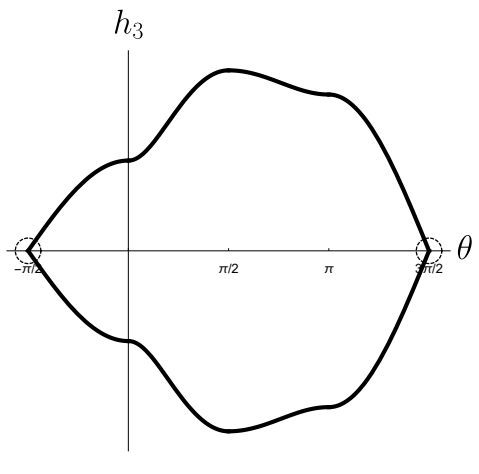

Figure 42: Mixed extremals $\left(\theta(t), h_{3}(t)\right)$ with $h_{4}>$ $h_{5}>0$

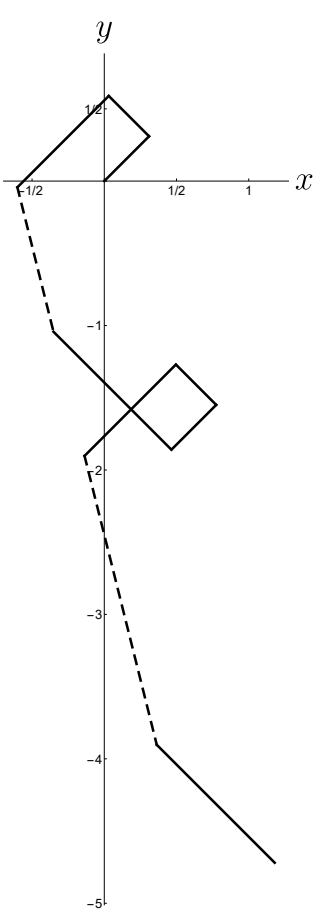

Figure 43: Example of mixed trajectory $(x(t), y(t))$ with $h_{4}>h_{5}>0$ 


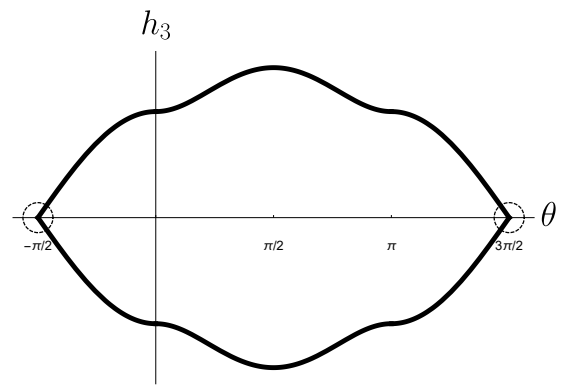

Figure 44: Mixed extremals $\left(\theta(t), h_{3}(t)\right)$ with $h_{4}>$ $h_{5}=0$

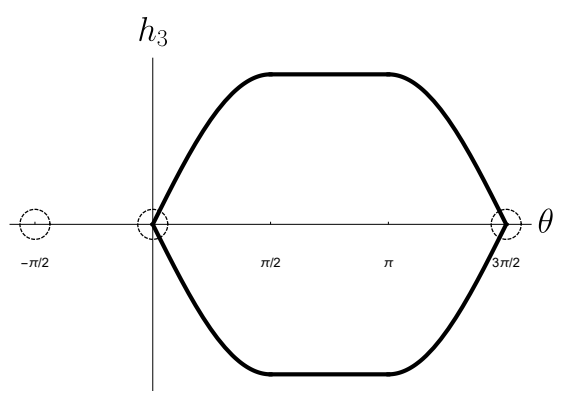

Figure 46: Mixed extremals $\left(\theta(t), h_{3}(t)\right)$ with $h_{4}=$ $h_{5}>0$

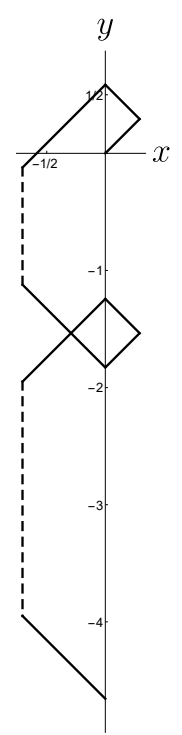

Figure 45: Example of mixed trajectory $(x(t), y(t))$ with $h_{4}>h_{5}=0$

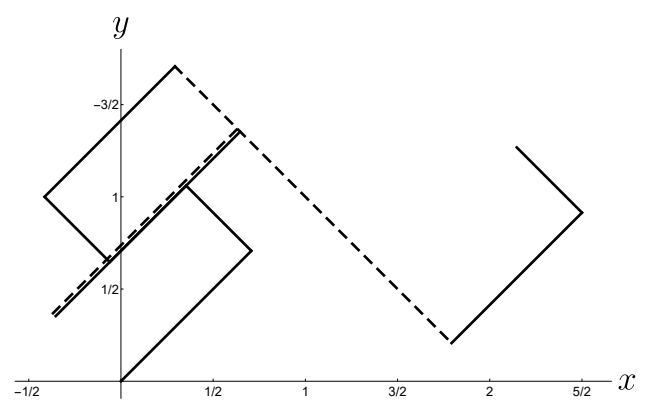

Figure 47: Example of mixed trajectory $(x(t), y(t))$ with $h_{4}=h_{5}>0$

Notice that singular arcs, unlike bang-bang ones, may have arbitrary durations.

Mixed extremals for $h_{4} \geq h_{5} \geq 0$ arise in the following cases:

- Case 1): $h_{4}>h_{5}>0, \theta=\frac{3 \pi}{2}, h_{3}=0$, level line $C_{6}$, see Figs. 42,43 ,

- Case 2): $h_{4}>h_{5}=0, \theta=\frac{3 \pi}{2}, h_{3}=0$, level line $C_{5}$, see Figs. 44 , 45 ,

- Case 3): $h_{4}=h_{5}>0, \theta \in\left\{0, \frac{3 \pi}{2}\right\}, h_{3}=0$, level line $C_{2}$, see Figs. 46,47

Theorem 3 yields the following bound.

Theorem 8. Optimal mixed controls have not more that 13 switchings.

Notice that mixed extremals $\lambda_{t}$ are not uniquely determined by the initial covector $\lambda_{0}$ and time $t$, because of arbitrary duration of singular arcs. Thus exponential mapping cannot be defined for mixed extremals, as it was defined for bang-bang ones.

\section{Bound on the number of arcs of minimizers}

Important questions for applications of sub-Finsler geometry in metric group theory are the following:

- given any pair of points in a sub-Finsler manifold, does there exist a piecewise-smooth minimizer that connects these points? 
- is there a uniform bound on the number of smooth arcs for piecewise smooth minimizers that connect arbitrary points in the manifold?

On the basis of our results we can provide affirmative answer for the both questions for the $\ell_{\infty}$ sub-Finsler problem studied in this paper.

Corollary 2. Any two points in the Cartan group can be connected by a piecewise smooth minimizer with not more than 14 smooth arcs.

Proof. By Th. 7, any two points in the Cartan group can be connected by a minimizer that belongs to the following (mutually not excluding) classes:

1. abnormal,

2. singular,

3. bang-bang,

4. mixed.

Abnormal trajectories are smooth (see Th. 2 [17]). If two points can be connected by a singular trajectory, then they can be connected by a piecewise smooth singular trajectory with not more than 5 smooth arcs (see Prop. 1 [17]). Bang-bang minimizers are piecewise smooth with up to 12 smooth arcs (see Th. 6). Finally, mixed minimizers are piecewise smooth with up to 14 smooth arcs (see Th. 8).

Moreover, we can now prove Th. 1 as a corollary of previously obtained results.

Proof. Classification of minimizers into types $(i)-(i i i)$ follows from Th. 7. The bound on the number of switchings on minimizers of type $(i i)$ and not of type $(i)$ is given by Th. 6. a similar bound for type (iii) is obtained by Th. 8 . The length-minimizing property of trajectories of type $(i)$ follows from Lemma 2 [17. Existence of a piecewise smooth minimizer with up to 5 smooth arcs for every trajectory of type $(i)$ follows from Prop. 1 [17.

\section{Conclusion}

In this paper we continued a study of the $\ell_{\infty}$ sub-Finsler problem on the Cartan group. Many questions remain unsolved, e.g.:

- cut time along bang-bang and mixed trajectories,

- cut locus,

- regularity of sub-Finsler distance and sphere.

We postpone study of these questions to forthcoming papers.

\section{Acknowledgments}

The authors thank Prof. Andrei Agrachev and Prof. Lev Lokutsievsky for fruitful discussions of sub-Finsler geometry.

\section{References}

[1] Ugo Boscain, Thomas Chambrion, and Grégoire Charlot, Nonisotropic 3-level quantum systems: complete solutions for minimum time and minimum energy, Discrete Contin. Dyn. Syst. Ser. B 5 (2005), no. 4, 957-990 (electronic).

[2] Valerii N. Berestovskii, Homogeneous manifolds with an intrinsic metric. II, Sibirsk. Mat. Zh. 30 (1989), no. 2, $14-28,225$.

[3] Valerii N. Berestovskii, The structure of locally compact homogeneous spaces with an intrinsic metric, Sibirsk. Mat. Zh. 30 (1989), no. 1, 23-34. 
[4] Emmanuel Breuillard and Enrico Le Donne, On the rate of convergence to the asymptotic cone for nilpotent groups and subFinsler geometry, Proc. Natl. Acad. Sci. USA 110 (2013), no. 48, 19220-19226.

[5] Jeanne N. Clelland and Christopher G. Moseley, Sub-Finsler geometry in dimension three, Differential Geom. Appl. 24 (2006), no. 6, 628-651.

[6] Michael G. Cowling and Alessio Martini, Sub-Finsler geometry and finite propagation speed, Trends in harmonic analysis, Springer INdAM Ser., vol. 3, Springer, Milan, 2013, pp. 147-205.

[7] Jeanne N. Clelland, Christopher G. Moseley, and George R. Wilkens, Geometry of sub-Finsler Engel manifolds, Asian J. Math. 11 (2007), no. 4, 699-726.

[8] Eero Hakavuori and Enrico Le Donne, Blowups and blowdowns of geodesics in carnot groups, Preprint, submitted, arXiv:1806.09375 (2018).

[9] Enrico Le Donne, A metric characterization of Carnot groups, Proc. Amer. Math. Soc. 143 (2015), no. 2, 845-849.

[10] Pierre Pansu, Métriques de Carnot-Carathéodory et quasiisométries des espaces symétriques de rang un, Ann. of Math. (2) 129 (1989), no. 1, 1-60.

[11] Yu. L. Sachkov, Exponential mapping in generalized Dido's problem, Mat. Sbornik, 194 (2003), 9: 63-90 (in Russian). English translation in: Sbornik: Mathematics, 194 (2003).

[12] A.A. Agrachev, Yu. L. Sachkov, Control Theory from the Geometric Viewpoint, Springer-Verlag, Berlin 2004.

[13] L.S. Pontryagin, V.G. Boltyanskii, R.V. Gamkrelidze, E.F. Mishchenko, The mathematical theory of optimal processes, Wiley Interscience, 1962.

[14] A. A. Agrachev and R. V. Gamkrelidze, Symplectic geometry for optimal control, Nonlinear controllability and optimal control, Monogr. Textbooks Pure Appl. Math., vol. 133, Dekker, New York, 1990, pp. 263- 277.

[15] Davide Barilari, Ugo Boscain, Enrico Le Donne, Mario Sigalotti, Sub-Finsler Structures from the Time-Optimal Control Viewpoint for some Nilpotent Distributions, J. Dyn. Control Syst. (2017) 23: 547.

[16] F. R. Gantmacher, The Theory of Matrices, Chelsea Pub. Co., 1960.

[17] A.Ardentov, E. Le Donne, Yu. Sachkov, A sub-Finsler problem on the Cartan group, Proceedings of Steklov Institute of Mathematics, 2019, accepted. 